\title{
Tuba Activates Cdc42 during Neuronal Polarization Downstream of the Small GTPase Rab8a
}

\author{
Pamela J. Urrutia, ${ }^{1,3}{ }^{\circ}$ Felipe Bodaleo, ${ }^{1,3}$ Daniel A. Bórquez, ${ }^{4}$ Yuta Homma, ${ }^{6}$ Victoria Rozes-Salvador, ${ }^{5}$ \\ Cristopher Villablanca, ${ }^{1,3}$ Cecilia Conde, ${ }^{5}$ Mitsunori Fukuda, ${ }^{6}$ and ${ }^{\circledR}$ Christian González-Billault ${ }^{1,2,3,7}$ \\ ${ }^{1}$ Laboratory of Cell and Neuronal Dynamics, Department of Biology, Faculty of Sciences, Universidad de Chile, 7800003, Santiago, Chile, \\ ${ }^{2}$ Department of Neuroscience, Faculty of Medicine, Universidad de Chile, 8380447, Santiago, Chile, ${ }^{3}$ Geroscience Center for Brain Health and \\ Metabolism, 7800003, Santiago, Chile, ${ }^{4}$ Laboratory of Signalling and Bioinformatics, Faculty of Medicine, Universidad Diego Portales, 8370007, \\ Santiago, Chile, ${ }^{5}$ Instituto de Investigación Médica Mercedes y Martin Ferreyra, Consejo Nacional de Investigaciones Científicas y Técnicas, \\ Universidad Nacional de Córdoba, X5016NST, Córdoba, Argentina, ${ }^{6}$ Laboratory of Membrane Trafficking Mechanisms, Department of Integrative \\ Life Sciences, Graduate School of Life Sciences, Tohoku University, 980-8577, Sendai, Japan, and ${ }^{7}$ The Buck Institute for Research on Aging, 94945 , \\ Novato
}

The acquisition of neuronal polarity is a complex molecular process that depends on changes in cytoskeletal dynamics and directed membrane traffic, regulated by the Rho and Rab families of small GTPases, respectively. However, during axon specification, a molecular link that couples these protein families has yet to be identified. In this paper, we describe a new positive feedback loop between Rab8a and Cdc42, coupled by Tuba, a Cdc42-specific guanine nucleotide-exchange factor (GEF), that ensures a single axon generation in rodent hippocampal neurons from embryos of either sex. Accordingly, Rab8a or Tuba gain-of-function generates neurons with supernumerary axons whereas Rab8a or Tuba loss-of-function abrogated axon specification, phenocopying the well-established effect of Cdc42 on neuronal polarity. Although Rab8 and Tuba do not interact physically, the activity of Rab8 is essential to generate a proximal to distal axonal gradient of Tuba in cultured neurons. Tuba-associated and Rab8a-associated polarity defects are also evidenced in vivo, since dominant negative (DN) Rab8a or Tuba knock-down impairs cortical neuronal migration in mice. Our results suggest that Tuba coordinates directed vesicular traffic and cytoskeleton dynamics during neuronal polarization.

Key words: axon specification; cytoskeleton; neuronal differentiation; neuronal polarity; Rab GTPases; Rho GTPases

Significance Statement

The morphologic, biochemical, and functional differences observed between axon and dendrites, require dramatic structural changes. The extension of an axon that is $1 \mu \mathrm{m}$ in diameter and grows at rates of up to $500 \mu \mathrm{m} / \mathrm{d}$, demands the confluence of two cellular processes: directed membrane traffic and fine-tuned cytoskeletal dynamics. In this study, we show that both processes are integrated in a positive feedback loop, mediated by the guanine nucleotide-exchange factor (GEF) Tuba. Tuba connects the activities of the Rab GTPase Rab8a and the Rho GTPase Cdc42, ensuring the generation of a single axon in cultured hippocampal neurons and controlling the migration of cortical neurons in the developing brain. Finally, we provide compelling evidence that Tuba is the GEF that mediates Cdc42 activation during the development of neuronal polarity.

Received Mar. 17, 2020; revised Dec. 21, 2020; accepted Dec. 29, 2020.

Author contributions: C.G.-B. and P.J.U. designed research; F.B., P.J.U., V.R.-S., D.A.B., and C.V. performed research; M.F. and Y.H. contributed unpublished reagents/analytic tools; C.G.-B., F.B., P.J.U., V.R.-S., C.C., D.A.B., C.V., M.F., and Y.H. analyzed data; C.G.-B. wrote the paper.

This work was supported by Comisión Nacional de Investigación Científica y Tecnológica grants to C.G.-B. under the Fondo Nacional de Ciencia y Tecnología (Fondecyt) (\#1180419) and Fondo Nacional de Áreas Prioritarias (FONDAP) (\#15150012) programs. P.U. was supported by the Fondecyt (\#3160630) Postdoctoral program. C.G.-B. and C.C. were supported by the International Brain Research Organization (IBRO) PROLAB grant. We thank Dr. Michael Handford for language support and Cristina Olmos and Kazuyasu Shoji for technical assistance.

The authors declare no competing financial interests.

Correspondence should be addressed to Christian González-Billault at chrgonza@uchile.cl.

https://doi.org/10.1523/JNEUROSCI.0633-20.2020

Copyright $\odot 2021$ the authors

\section{Introduction}

Neurons are highly polarized cells, in which sprouting and elongation of neurites, and their subsequent development into axons and dendrites are key events during early neuronal differentiation (Dotti et al., 1988; Bradke and Dotti, 2000; Barnes and Polleux, 2009). These cellular processes are predominantly dependent on changes in cytoskeletal dynamics and vectorially directed membrane traffic, controlled by the Rho and Rab families of small GTPases respectively, that provide the driving force for neurite elongation and growth cone pathfinding and turning (Dotti et al., 1988; Arimura and Kaibuchi, 2007; Cheng and Poo, 2012; Lalli, 2014). The small Rho GTPase Cdc42, has emerged as 
a critical regulator of neuronal polarity, allowing the activation of the PAR polarity complex, composed of an atypical protein kinase $\mathrm{C}(\mathrm{aPKC})$ isoform and the scaffolding proteins Par3 and Par6. This complex accumulates at the tips of axons, promoting axon specification via actin filament remodeling (Shi et al., 2003; Nishimura et al., 2005; Lalli, 2009). While it is widely accepted that $\mathrm{Cdc} 42$ activation is required to drive axon specification, the identity of the guanine nucleotide-exchange factor (GEF) responsible for such activation during the acquisition of neuronal polarity is still unknown.

Many other cell types are also highly polarized, including epithelial cells. Their polarization mechanism has been extensively described and shares several similarities with neuronal polarization (Bonifacino, 2014). Tuba, also named dynamin-binding protein (DNMBP), a multidomain scaffold protein with GEF activity specific for $\mathrm{Cdc} 42$, is essential to drive epithelial cell polarization (Bryant et al., 2010; Pichaud et al., 2019). Tuba is highly concentrated at neuronal synapses, where it interacts with dynamin to regulate the actin cytoskeleton (Salazar et al., 2003). Tuba-mediated actin cytoskeleton regulation involves the activation of Cdc42 and its binding with several other proteins, including several actin dynamic regulators such as neural WiskottAldrich syndrome protein (N-WASP), WAS/WASL-interacting protein family member 3 (WIPF3), WASP-family verprolin homologous protein 1 (WAVE1), WASP binding protein (WIRE), cytoplasmic FMR1-interacting protein 2 (CYFIP2), nucleosome assembly protein 1 (NAP1), and enabled/vasodilator-stimulated phosphoprotein (Ena/VASP; Salazar et al., 2003; Cestra et al., 2005; Kovacs et al., 2011). Moreover, Tuba plays a significant role in long-term memory formation (Casoli et al., 2012). However, a role in neuronal polarization has not been reported to date.

On other hand, it has been recently described that exocytic (secretory) and endocytic pathways are critical for neuronal differentiation and axonal elongation (Villarroel-Campos et al., 2014, 2016a,b). In the secretory pathway, the small Rab GTPase Rab8 regulates the transport of exocytic vesicles from the trans-Golgi network (TGN) to the plasma membrane (Stenmark, 2009). In embryonic hippocampal neurons, Rab8 deficiency impairs axon formation and decreases neurite outgrowth, diminishing anterograde movement of vesicles that accumulate in the Golgi apparatus (Huber et al., 1995). Conversely the overexpression of a constitutively active (CA) mutant of Rab8a increases axonal outgrowth in mice cortical neurons (Furusawa et al., 2017). Remarkedly, Rab8 controls apical Cdc42 activation through Tuba, ensuring the correct epithelial polarization (Bryant et al., 2010). Therefore, here we evaluated whether Tuba is also the GEF that activates Cdc42 downstream of Rab8a during axon specification. We report that Tuba regulates neuronal polarity by activation of Cdc42, through a positive feedback loop between Rab8a and Cdc42. Altogether, these findings suggest that Tuba may be one of the linkers that connects the regulation of cytoskeletal dynamics and the directed vesicular traffic during neuronal polarization.

\section{Materials and Methods}

\section{Primary culture}

Neuronal hippocampal cultures were prepared as previously described (Kaech and Banker, 2006). Briefly, hippocampi were dissected from Embryonic day 18 (E18) Sprague Dawley rat embryos or Embryonic day 17 (E17) C57BL/6 mice embryos of either sex, and treated with $0.25 \%$ trypsin in HBSS (Invitrogen) for $25 \mathrm{~min}$ at $37^{\circ} \mathrm{C}$. The tissue was mechanically-dissociated, and neurons were seeded onto sterilized glass coverslips coated with poly-L-lysine $(1 \mathrm{mg} / \mathrm{ml}$, Sigma-Aldrich) in DMEM (Invitrogen) containing 10\% horse serum, 1\% penicillin/streptomycin, and $1 \%$ glutamine (Invitrogen) for $1 \mathrm{~h}$. The medium was then replaced with neurobasal medium containing 2\% B27 (Invitrogen), 1\% Glutamax (Invitrogen), and 1\% penicillin/streptomycin (maintenance medium).

For nucleofection experiments, neurons in suspension were electroporated using the $4 \mathrm{D}$-nucleofector system before plating following the manufacturer's instructions (Lonza). Untagged constructs were conucleofected with a GFP-coding plasmid at a 4:1 ratio to ensure a $98 \%$ co-transfection rate, according to internal controls (data not shown).

\section{Cell culture}

The neuroblastoma N1E-115 cell line was obtained from the American Type Culture Collection (ATCC; CRL-2263). Cells were cultured in complete DMEM (Invitrogen) containing 5\% heat-inactivated fetal bovine serum (FBS) and $1 \%$ penicillin/streptomycin (Invitrogen) at $37^{\circ} \mathrm{C}$ and $5 \% \mathrm{CO}_{2}$. Cells were transfected in serumfree DMEM with Lipofectamine 2000 (Invitrogen) according to the manufacturer's instructions. The culture medium was replaced with fresh medium at $4 \mathrm{~h}$ after transfection, and cells were processed 24$48 \mathrm{~h}$ later.

\section{Plasmids}

The Raichu-Cdc42 Förster resonance energy transfer (FRET) biosensor probe was obtained from A. Cáceres (Instituto de Investigación Médica Mercedes y Martín Ferreyra). Rab8aWT-GFP, Rab8aQ67LGFP, Rab8aT22N-GFP, Rab8bWT-GFP, Rab8bQ67L-GFP, Rab8bT22NGFP, Rab8aWT-Flag, Rab8aQ67L-Flag, Rab8aT22N-Flag, Rab8bQ67L-Flag, Rab8bQ67L-Flag, and Rab8bT22N-Flag (GFP and Flag are N-terminally tagged) plasmids were prepared as described previously (Fukuda, 2003; Homma and Fukuda, 2016). shRNAs against rat Rab8a cloned into the pRFP-C-RS vector (TF712324, locus ID 117103), shRNAs against rat Rab8b cloned into the pGFP-V-RS vector (TG713207, locus ID 266688) and shRNAs against mouse DNMBP cloned into the pRFP-C-RS vector (TF515449, locus ID 71972) were purchased from OriGene. Cdc42F28LHA was provided by Richard A. Cerione (Cornell University, Ithaca, NY) and Cdc42-myc was purchased from Addgene.

\section{Antibodies}

The following primary antibodies were used in this study: mouse anti$\alpha$-tubulin (clone DM1A, T6199; Sigma-Aldrich), rabbit anti-MAP2 (AB5622, Millipore), rabbit anti-Rab8 (D22D8, Cell Signaling), rabbit anti-Tuba (AB154836, Abcam), mouse anti-tau-1 (MAB3420, Millipore), mouse anti- $\beta$ III-tubulin (G712A; Promega), rabbit anti-Cdc42 (07-1466; Millipore), mouse anti-cofilin, rabbit anti-phospho-cofilin (Ser3; a kind gift from J. R. Bamburg, Colorado State University, CO), rabbit anti-GFPHRP (598-7, MBL), and mouse anti-FLAG-HRP (A8592, Sigma-Aldrich). Secondary antibodies for immunoblots were HRP-conjugated anti-mouse and anti-rabbit IgG (The Jackson Laboratory). Secondary antibodies for immunocytochemistry were anti-mouse and anti-rabbit conjugated to Alexa Fluor 488, 543, or 633 (Thermo Fischer)

\section{Cdc42 activity pull-down assay}

The CRIB domain for Cdc42 activity was purified as described previously (Villarroel-Campos et al., 2016b). Briefly, loaded beads were incubated for $70 \mathrm{~min}$ at $4^{\circ} \mathrm{C}$ with $1.5 \mathrm{mg}$ of either a control or Cdc42expressing N1E-115 cell lysate using fishing buffer $(50 \mathrm{~mm}$ Tris- $\mathrm{HCl}, \mathrm{pH}$ 7.5, 10\% glycerol, $1 \%$ Triton X-100, $200 \mathrm{~mm} \mathrm{NaCl}, 10 \mathrm{~mm} \mathrm{MgCl}_{2}, 25 \mathrm{~mm}$ $\mathrm{NaF}$, and protease inhibitor cocktail). The beads were washed three times with washing buffer (50 mM Tris- $\mathrm{HCl}, \mathrm{pH} 7.5,30 \mathrm{mM} \mathrm{MgCl}, 40$ $\mathrm{mm} \mathrm{NaCl}$ ) and then resuspended in SDS-PAGE sample buffer. Bound Cdc42-GTP was subjected to immunoblot analysis and quantified with respect to total Cdc42 using ImageJ.

Cdc42 activity FRET assay

To measure GTPase activity, neurons were co-transfected with FlagRab8a (Q67L or T22N) and Raichu-Cdc42 FRET biosensor for $24 \mathrm{~h}$. FRET efficiency measurements were performed as described previously (Nakamura et al., 2006). Briefly, transfected neurons were excited at 
$450 \mathrm{~nm}$, and emissions were collected at 460-490 and 505-530 nm (donor and acceptor emission wavelengths, respectively). The ratio of the acceptor-to-donor emission was established as the FRET efficiency. The FRET map was generated by dividing the acceptor-to-donor ratio image by the binary mask of the same image. Measurement of FRET efficiency was performed by selecting a region of interest at the soma, and at the proximal or distal axon.

\section{Immunoprecipitation}

COS-7 cells were transfected with plasmids encoding FLAG-Rab8A or EGFP-Tuba. Cells were lysed with a lysis buffer $(50 \mathrm{~mm}$ Tris- $\mathrm{HCl}, \mathrm{pH}$ 7.5, $150 \mathrm{~mm} \mathrm{NaCl}, 1 \%$ Triton X-100, 10\% glycerol, $100 \mathrm{~mm} \mathrm{MgCl}, 1 \mathrm{~mm}$ dithiothreitol, and $1 \times$ protease inhibitor; Roche, \#1873580) and centrifuged at $17,400 \times g$ for $10 \mathrm{~min}$ at $4^{\circ} \mathrm{C}$ to remove insoluble materials. FLAG-Rab8A was immunoprecipitated using anti-FLAG agarose beads (Sigma-Aldrich) and loaded with either GppNHp (a non-hydrolyzable GTP analog) or GDP, and then they were incubated with the lysate of EGFP-Tuba-expressing cells for $2 \mathrm{~h}$ at $4^{\circ} \mathrm{C}$. The beads were washed with the lysis buffer three times and boiled in a sample buffer $(62.5 \mathrm{~mm}$ Tris$\mathrm{HCl}, \mathrm{pH} 6.8,2 \%$ 2-mercaptoethanol, 2\% SDS, $10 \%$ glycerol, and $0.2 \%$ bromophenol blue). The samples were subjected to SDS-PAGE and analyzed by immunoblotting with appropriate antibodies.

\section{Immunoblotting}

Protein extracts were obtained from cell lines or primary neuronal cultures. Cells were lysed with radioimmunoprecipitation assay (RIPA) buffer, subjected to SDS-PAGE and electroblotted onto nitrocellulose or polyvinylidene difluoride membranes. Blots were probed with the appropriate primary and secondary antibodies, and immunoreactivity signals were visualized with an enhanced chemiluminescent substrate (Thermo Scientific) and quantified by densitometry using ImageJ.

\section{Immunocytochemistry}

Cultures were fixed with $4 \%$ paraformaldehyde (PFA)/4\% sucrose in PBS for $30 \mathrm{~min}$ at $37^{\circ} \mathrm{C}$, washed three times with PBS and then permeabilized with $0.2 \%$ Triton X-100 in PBS. Then coverslips were blocked with $5 \%$ BSA in PBS, primary antibodies were added in $1 \%$ BSA in PBS, and cells were incubated overnight at $4^{\circ} \mathrm{C}$. Cells were washed three times with PBS and incubated with the appropriate Alexa Fluor-conjugated secondary antibodies for $1 \mathrm{~h}$ at room temperature (RT). Coverslips were mounted in FluorSave (Millipore) and analyzed using a confocal microscope (Zeiss LSM 810). Axon length was measured using the microscope-associated software, LSM Image Browser (Zeiss). Confocal images presented here were color-inverted to improve morphologic appreciation.

\section{In utero electroporation (IUE) and imaging acquisition}

IUE were conducted following previous reports (Fuentes et al., 2012; Mestres et al., 2016). Briefly, pregnant E15 C57BL/6 mice were anesthetized with isofluorane/oxygen mix ( $4 \%$ for induction and $2 \%$ for maintenance) during the whole surgery, and Tramadol $(5 \mathrm{mg} / \mathrm{Kg})$ was used as analgesia during the procedure. Uterine horns were exposed, and embryos were locally injected with pCAG-GFP, pCAGGFP+Rab8aQ67L-GFP, pCAG-GFP+Rab8aT22N-GFP, pRFP-shscramble, or pRFP-sh-Tuba into the lateral ventricle of the brain. To visualize successful injections, the fast green FCF dye (Sigma-Aldrich, catalog \#F7252) was co-injected with DNAs. Then, brains were electroporated using a BTX electroporator $(\Delta \mathrm{V}=39 \mathrm{~V}$; pulses: 5; duration: $50 \mathrm{~ms}$; intervals between pulses: $950 \mathrm{~ms}$ ) with Tweezers w/Variable Gap 2 Square Platinum Electrodes (Nepagene, CUY647P2X2). After electroporation, in utero embryos were returned to the maternal cavity to recover from the surgery. At E18, embryos were killed to check GFP or RFP expression in control and Rab8aT22N or Rab8aQ67L genetic contexts. Brains expressing GFP or RFP were fixed overnight in $4 \% \mathrm{w} / \mathrm{v}$ PFA solution dissolved in $\mathrm{PBS}$ at $4^{\circ} \mathrm{C}$ with gentle agitation. Then, fixed brains were immersed into a $30 \% \mathrm{w} / \mathrm{v}$ sucrose solution for $24 \mathrm{~h}$ at $4^{\circ} \mathrm{C}$. Postfixed brains were frozen at $-25^{\circ} \mathrm{C}$ using Crioplast solution (Biopack). The cerebral cortex was sliced into $50-\mu \mathrm{m}$ cortical sections using a cryostat (Leica CM 1850). Brain slices were mounted onto glass slides. Tissues were permeabilized with $0.3 \% \mathrm{v} / \mathrm{v}$ Triton X$100-$ PBS solution, followed by DAPI staining ( $15 \mathrm{~min}$ at RT). Then, samples were mounted in Mowiol solution (Sigma) for z-stack imaging in a Zeiss LSM 810 confocal microscopy. Images were acquired with a $20 \times$ air objective. Several fields were acquired to image the whole brain cortex [from the ventricular zone (VZ) to the cortical plate $(\mathrm{CP})$ ]. Collected images were stitched using the Stitching plugin of Fiji-ImageJ.

\section{Statistical analyses}

All data represent mean \pm SEM of at least three independent experiments. Comparisons between two groups were made using unpaired Student's $t$ tests when data presented a Gaussian distribution, and nonparametric Mann-Whitney tests when data presented a non-Gaussian distribution. Comparisons between more than two groups were conducted using one-way ANOVA followed by Bonferroni's post hoc test. A value of $p<0.05$ was considered significant.

\section{Results}

Tuba, a Cdc42 GEF, is required for axon specification during neuronal polarization

Tuba is expressed in adult brain and co-localizes with synaptic markers (Salazar et al., 2003). Two isoforms of Tuba have been described, Tuba full-length (TubaFL; $\sim 180 \mathrm{kDa}$ ) and miniTuba (mTuba; $\sim 75 \mathrm{kDa}$ ), differing by the inclusion (by alternative splicing) of four N-terminal SH3 (Src-homology 3) domains (Salazar et al., 2003). Considering its role in epithelial cell polarity, we first analyzed the expression, distribution, and function of Tuba in primary cultures of embryonic neurons. We detected increasing levels of TubaFL (using an isoform-specific antibody) during first $3 \mathrm{~d}$ in vitro (DIV), when the axon specification occurs (Fig. 1A). Immunofluorescence staining showed a punctate pattern with perinuclear distribution in stage 1 neurons. In unpolarized stage 2 neurons, Tuba accumulated in a single minor neurite and became enriched in the axonal hillock and axonal growth cone on polarization (stage 3; Fig. 1B). To define whether Tuba is involved in axon formation, we performed knock-down experiments in cultured hippocampal neurons using an shRNA against Tuba. The efficiency of the shRNA construct was tested by Western blotting in N1E-115 neuroblastoma cells (data not shown). Mouse hippocampal neurons were nucleofected before plating with the shRNA-encoding vector and analyzed at 3 DIV by immunofluorescence staining against MAP2 and tau1, as dendritic and axonal markers, respectively (Fig. 1C). Tuba knockdown increased the number of unpolarized neurons compared with scrambled shRNA-transfected neurons $(7.35 \pm 3.47 \%$ vs $40.98 \pm 6.6 \%$; Fig. 1D). In addition, Tuba knock-down generate a significant decrease in axonal length as compared with control neurons (Fig. $1 F$ ), without altering minor neurite mean length (Fig. 1E). These results indicate that Tuba is required for axon formation. In order to confirm the role of Tuba in neuronal polarization, we nucleofected TubaFL, mTuba or mTuba without the GEF domain (mTuba $\triangle \mathrm{GEF}$ ) constructs in hippocampal neurons before plating and analyzed at 3 DIV using immunofluorescence staining against MAP2 and tau-1 (Fig. 1G). TubaFL nucleofection increased the number of neurons showing multiple axons $(12.77 \pm 1.68 \%$ vs $37.5 \pm 4.7 \%)$, whereas mTuba did not. Similar to Tuba knock-down, mTuba $\Delta$ GEF nucleofection increased the number of unpolarized neurons $(6.77 \pm 4.39 \%$ vs $40.63 \pm 0.93 \%$; Fig. $1 H)$. That results suggest that $\mathrm{mTuba} \triangle \mathrm{GEF}$ acts in a dominant negative (DN) manner, as previously noted by Qin et al., in an epithelial polarity model (Qin et al., 2010). We also found an increase in mean minor neurite length in neurons transfected with mTuba, while 
A

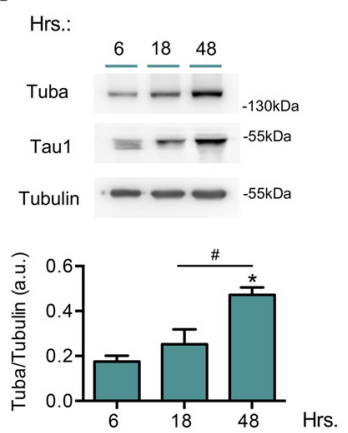

D

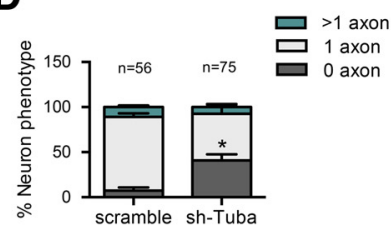

B

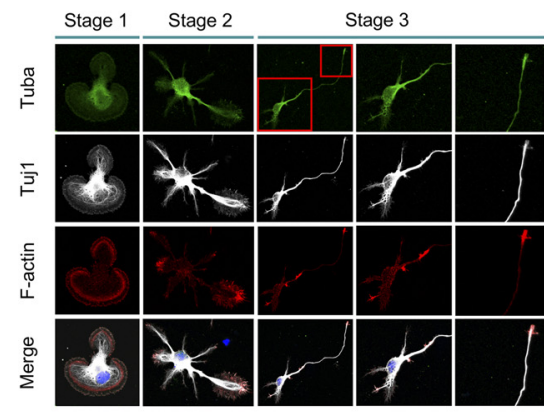

E

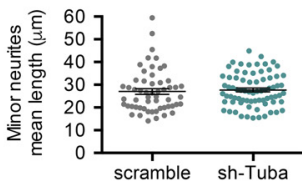

C

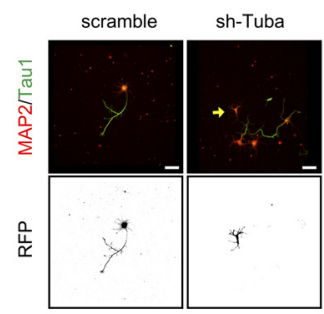

F

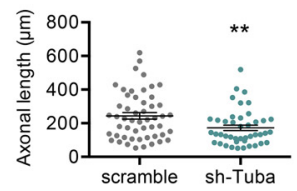

G

H
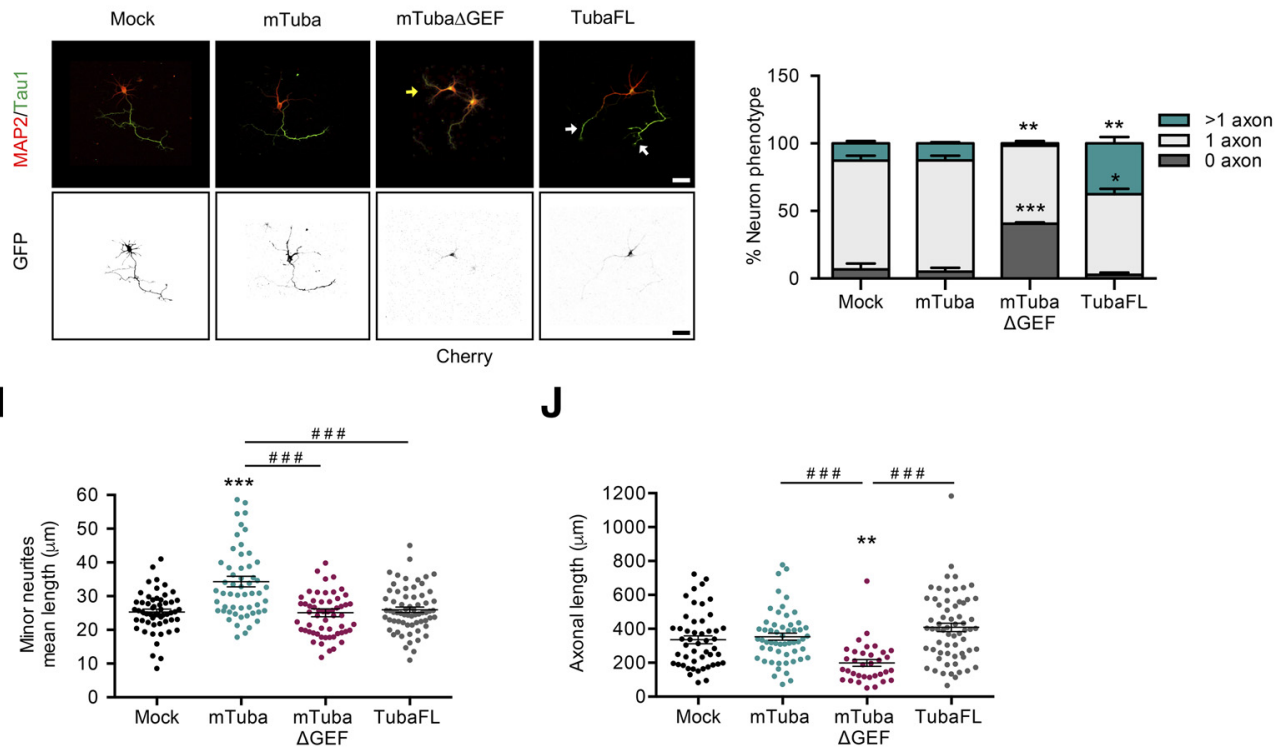

Figure 1. Tuba, a $\mathrm{Cd}(42 \mathrm{GEF}$, is required for axon specification during neuronal polarization. $A$, Representative Western blotting and quantification of Tuba expression from cultured cortical neurons at 6, 18, and $48 \mathrm{~h}$ postplating. $\boldsymbol{B}$, Representative images of neurons at stages 1-3 stained with Tuba (green), Tuj1 (gray), F-actin (red), and T0-PRO-3 (blue). C, Representative images of hippocampal neurons overexpressing scrambled or shRNA against Tuba, stained with MAP2 and tau-1. D, Quantification of neuronal phenotypes of RFP-positive cells in C. $\boldsymbol{E}$, Quantification of minor neurite average length in $\boldsymbol{C}$. $\boldsymbol{F}$, Quantification of axonal length in $\boldsymbol{C}$. $\boldsymbol{G}$, Representative images of hippocampal neurons overexpressing mock, mTuba, mTuba $\Delta G E F$, or TubaFL, stained with MAP-2 and tau-1. $\boldsymbol{H}$, Quantification of neuron phenotypes of GFP or Cherry-positive cells in $\mathbf{G}$. I, Quantification of mean minor neurite length in $\mathbf{G}$. $\boldsymbol{J}$, Quantification of axonal length in $\mathbf{G}$. Yellow arrow show nucleofected neurons and white arrow shows axons in multiaxonic neurons. Scale bars: $50 \mu$ m. Values represent mean \pm SEM $(n=3$ or 4$) ; * p<0.05, * * p<0.01$, $* * * p<0.001$ as compared with the corresponding control; $\# p<0.05$ and \#\#\#p<0.001 as compared between conditions.

TubaFL or mTuba $\triangle$ GEF nucleofection had not effect (Fig. $1 I$ ). Furthermore, we detected a reduction in the total axonal length in mTuba $\Delta$ GEF nucleofected neurons compared with control, but no differences in the total axonal length between TubaFL or mTuba expressing neurons (Fig. 1J), suggesting that the role of Tuba in axon formation is partially dependent on its GEF activity.

\section{Tuba axonal distribution is regulated by Rab8a in an} interaction-independent manner

TubaFL (and not mTuba) can interact with GM130, a peripheral membrane protein of the cis-Golgi stack, controlling Cdc42 activation at the Golgi apparatus (Kodani et al., 2009). As Rab8 was originally described as a trafficking regulator between the Golgi apparatus and the plasma membrane, we then evaluated the codistribution of Tuba and Rab8 with GM130. We performed immunofluorescence staining at 3 DIV in hippocampal neurons overexpressing TubaFL-GFP and immunostained against endogenous Rab8 and GM130. Tuba-GFP was used instead of Tuba immunostaining, because Tuba antibody was raised in the same species as GM130 antibody.

We observed that Rab8 and Tuba partially co-localized with the GM130-positive compartment and also at the axonal growth cone (Fig. 2A), with Pearson"s correlation coefficient of $0.28 \pm$ 0.07 and $0.17 \pm 0.044$, respectively (Fig. $2 B$ ). In addition, we evaluated direct interaction between Rab8a and Tuba through immunoprecipitation assay. The experiments were performed in presence of excess GDP, which locked Rab8a in their inactive 
A
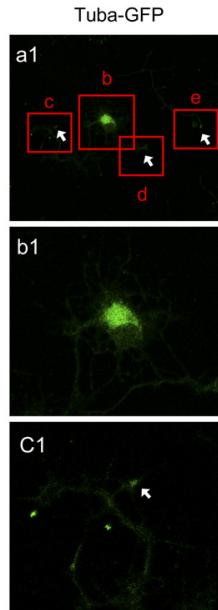

d1
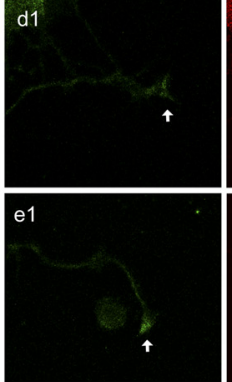

C

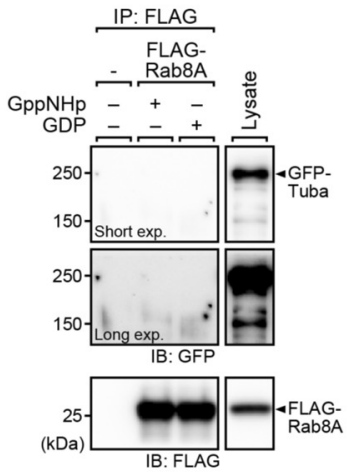

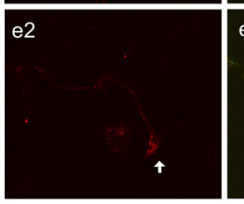

Rab8
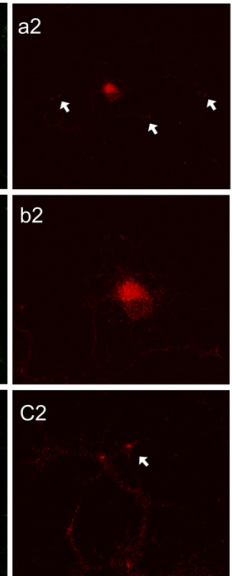

d2

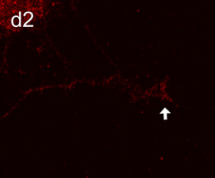

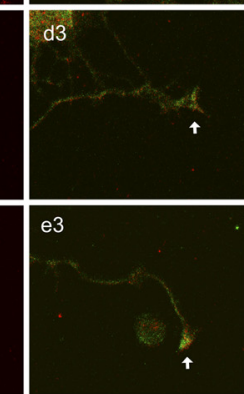

Rab8/Tuba-GFP
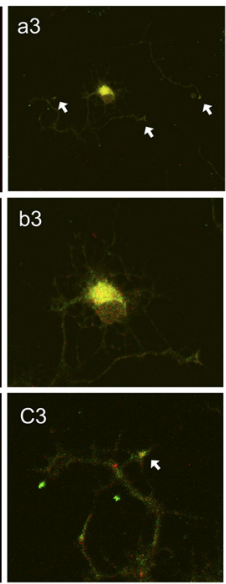

D

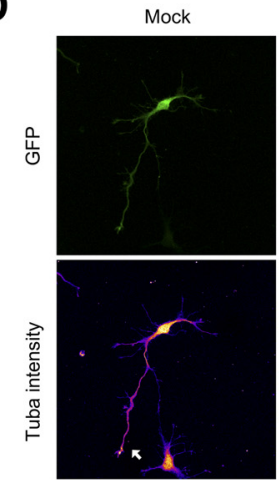

GM130

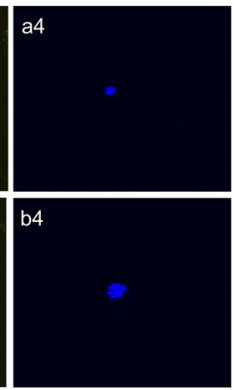

Tuba-GFP

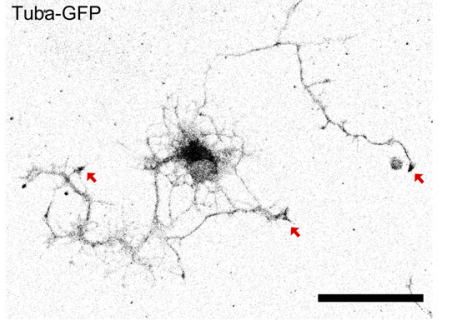

B
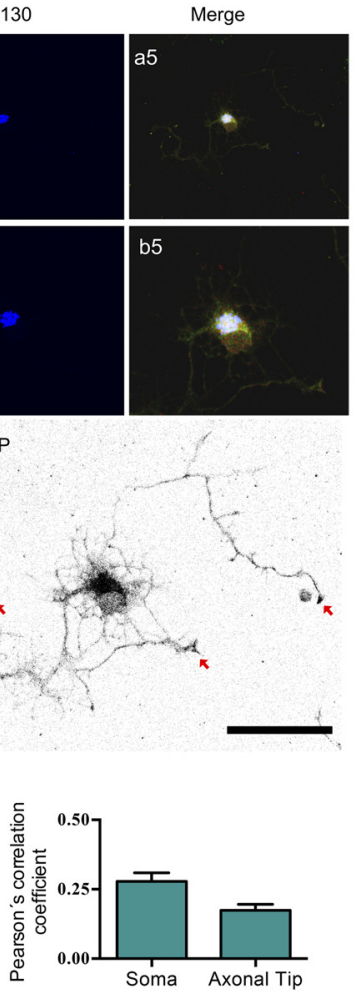

b5

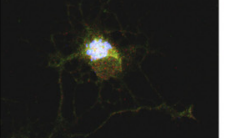

Rab8a Q67L

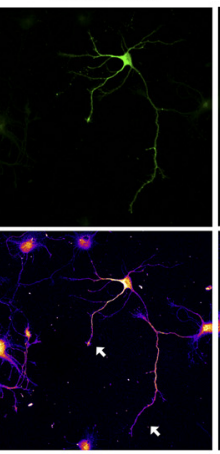

Rab8a T22N

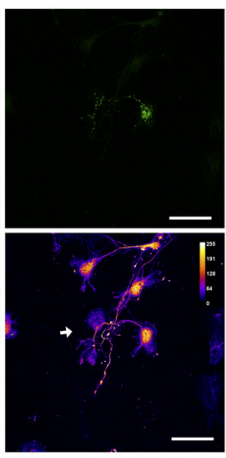

E

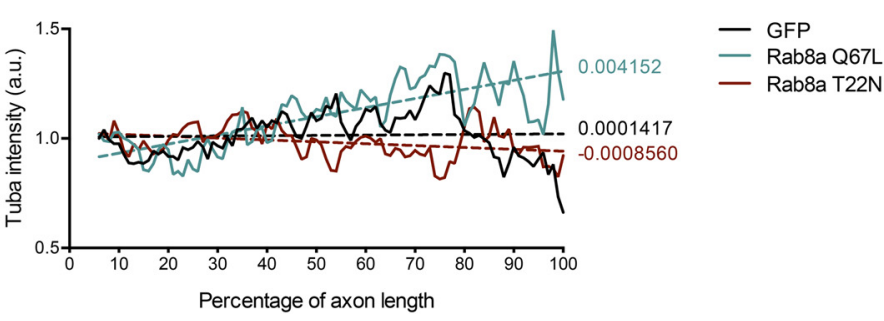

Figure 2. Tuba axonal distribution is regulated by Rab8a in an interaction independent manner. $\boldsymbol{A}$, Representative image of Tuba-transfected neurons stained with anti-rab8 (red) and antiGM130 (cis-Golgi marker, blue) antibodies. Columns 1-4 correspond to Tuba-GFP, Rab8, Rab8/Tuba-GFP, GM130, and merge, respectively. Rows $\boldsymbol{b}-\boldsymbol{e}$ correspond to a magnification indicated in row $\boldsymbol{a}$. A color-inverted TubaFL overexpressing neuron is shown on the lower right side of the panel. White arrow shows co-distribution between Rab8 and TubaFL-GFP, red arrow shows axons. $\boldsymbol{B}$, Quantification of Pearson's correlation coefficient in soma at neuronal tips of $\boldsymbol{A}$. C, FLAG-Rab8a expressed in COS-7 cells was immunoprecipitated and loaded with either GDP or GppNHp, and then FLAG-Rab8a-bound beads were incubated with the lysate of EGFP-Tuba-expressing cells. Blots were probed with an anti-GFP antibody to detect Tuba. $\boldsymbol{D}$, Representative images of hippocampal neurons overexpressing Rab8a Q67L (CA mutant) or Rab8a T22N (DN, mutant), showing a gradient of tuba intensity. White arrow shows axons (E) Quantification of Tuba intensity along percentage of axonal length of hippocampal neurons overexpressing Rab8a Q67L or Rab8a T22N. Scale bars: $50 \mu \mathrm{m}$.

conformation, or in the presence of a non-hydrolyzable GTP analog (GppNHp), which activates Rab8a. We observed that Tuba does not interact with Rab8a (Fig. 2C). Next, we evaluated whether Rab8a activity affects the distribution of Tuba along the axon. For that purpose, we nucleofected CA Rab8a Q67L and
DN Rab8a T22N in cultured hippocampal neurons and evaluated Tuba immunostaining along axons at 3 DIV. We observed that GFP-expressing control neurons have a homogeneous distribution of Tuba along the axon, showing a small local enrichment at the axonal hillock and growth cones (Fig. 2D). However, 
in Rab8a Q67L-transfected neurons, we appreciate an evident proximal-distal gradient of Tuba staining. In contrast Rab8a T22N-transfected neurons showed an axonal distribution that is more similar to GFP-expressing neurons (Fig. 2D). We quantified the mean Tuba fluorescence intensity along the axon for each experimental condition. We used a nonlinear regression with a $95 \%$ confidence interval for parameters, and we observed that Tuba fluorescence intensity along the axon in Rab8a Q67L-nucleofected neurons display a significant different slope compared with GFP or Rab8a T22Noverexpressing neurons. (Fig. 2E).

Therefore, these results suggest that although Rab8 and Tuba does not directly interact, but Rab8 activity promotes the enrichment of Tuba at the distal segment of the axon, possibly via a vesicular trafficking mechanism.

\section{Rab8 is required for axon formation}

To examine whether Rab8 is required for axon specification, we analyzed the protein expression, distribution, and function during early neuronal differentiation. In cultured neurons, we detected Rab8 expression $(\sim 25 \mathrm{kDa})$ as early as $6 \mathrm{~h}$ in vitro, which increased during neuronal polarization (Fig. 3A). Staining of cultured neurons showed a punctate pattern distributed on perinuclear region and minor neurites in unpolarized cells (stages 1 and 2) and in axons and minor neurites of polarized cells (stage 3; Fig. 3B). To determinate whether Rab8 was involved in axon formation, we performed knock-down experiments in cultured hippocampal neurons using an shRNA against Rab8. There are two Rab8 isoforms, termed Rab8a and Rab8b, which are encoded by different genes (Armstrong et al., 1996); therefore, we used isoform-specific shRNAs to distinguish their individual contribution to axonal development. The efficiency of each shRNA was assessed by Western blotting in transfectedB104 neuroblastoma cells (data not shown). Hippocampal neurons were then nucleofected before plating with shRNA against Rab8a and/or Rab8b and analyzed at 3 DIV using immunofluorescence staining against MAP2 and tau-1 (Fig. $3 C-E)$. Rab8a knock-down increased the number of unpolarized neurons $(7.36 \pm 1.68 \%$ vs $37.27 \pm 5.15 \%)$ compared with scrambled (Fig. 3F). Additionally, we analyzed the mean minor neurite length and total axonal length of nucleofected neurons at 3 DIV. Rab8a knock-down did not alter mean minor neurite length (Fig. $3 G$ ) but lead to a reduction in total axonal length (Fig. $3 H$ ). In contrast, Rab8b knock-down did not alter the number of unpolarized neurons or the mean minor neurite length (Fig. $3 D, F, G$ ) but reduced total axonal length (Fig. 3H). In addition, co-nucleofection using both shRNAs against Rab8a and Rab8b caused an increase in the percentage of unpolarized neurons (Fig. $3 E, F$ ), similarly to shRab8a alone. Moreover, Rab8a or Rab8a/b double knockdown reduces total axonal length (Fig. $3 H$ ) without alterations in a mean minor neurite length (Fig. $3 G$ ). Collectively, these results strongly suggest that Rab8a, but not Rab8b, is required for axon determination.

\section{Overexpression of a CA Rab8a isoform generates multiaxonal neurons}

To further analyze the role of Rab8a in axon specification, we nucleofected Rab8a and Rab8b WT, Rab8a and Rab8b Q67L (CA mutants), and Rab8a and Rab8b T22N (DN mutants) as GFP fusion proteins in cultured hippocampal neurons and addressed polarity acquisition by staining against MAP2 and tau- 1 at 3 DIV (Fig. 4A,E). The overexpression of Rab8a WT and Rab8a Q67L lead to a significant increase in the percentage of neurons showing multiple axons $(7.5 \pm 3.1 \%$ vs $30.69 \pm 2.3 \%$ and $58.88 \pm 4.8 \%$, respectively), whereas the overexpression of Rab8a T22N caused a significant increase in the number of unpolarized neurons $(4.53 \pm 4.53 \%$ vs $55.93 \pm 11.31 \%)$ compared with control conditions (Fig. 4B). In addition, we determined other morphometric parameters. Neurons overexpressing Rab8a Q67L showed no differences in the minor neurite length but had significant longer axons compared with controls. Neurons overexpressing Rab8a $\mathrm{T} 22 \mathrm{~N}$ displayed a significant decrease in both mean minor neurite length and axonal length compared with control (Fig. 4C,D).

In contrast, the overexpression of Rab8b WT or Rab8b Q67L generated no variations in the proportion of neurons showing multiple axons, although the overexpression of Rab8b T22N resulted in a significant increase in the number of unpolarized neurons (Fig. $4 F$ ). We did not observe differences in mean minor neurite length in neurons overexpressing Rab8b WT, Rab8b Q67L or Rab8b T22N compared with control (Fig. 4G). However, neurons overexpressing Rab8b T22N had significantly shorter axons compared with control neurons (Fig. $4 H$ ). These results clearly indicate that Rab8a is required for axon specification because diminished levels or the expression of a DN form abolished axon generation, whereas overexpression of the wild-type or a CA mutant induced the generation of supernumerary axons.

\section{Rab8a CA triggers Tuba-dependent Cdc42 activation}

It has been previously reported that Rab8 controls apical differentiation in epithelial cells by Cdc42 activation through recruitment of Tuba (Bryant et al., 2010). Therefore, we evaluated the effect of Rab8a activation on Cdc42 activity and the Serine three phosphorylation of cofilin, a readout of Cdc42 signaling pathway activation. Neuroblastoma N1E-115 cells were transfected with Rab8a WT, Rab8a Q67L or Rab8a T22N and Cdc42 activity was assessed by a pulldown assay. The overexpression of Rab8a Q67L induced a significant increase in Cdc42 activity and phosphocofilin compared with control (Fig. $5 A, B$ ). We also evaluated changes in Cdc42 activity in cultured primary neurons using a FRET-based approach. We expressed a Cdc42 FRET biosensor in conjunction with Rab8a Q67L or Rab8a T22N and measured pixel intensity in the resulting FRET maps in the somatodendritic, proximal axon or distal axon compartments (Fig. 5C). Rab8a Q67L overexpression significantly increases Cdc42 activity in all compartments compared with the control. No changes in Cdc42 activity were observed in neurons overexpressing Rab8a WT or Rab8a T22N (Fig. 5D,E). In addition, we evaluated the effect of the knock-down of Tuba on Cdc42 activation induced by Rab8a Q67L in a neuroblastoma cell line. We observed that shRNA against Tuba abrogated the increased Cdc42 activity induced by Rab8a Q67L (Fig. 5G). We also analyzed the effect of shRNA against Tuba in the morphologic changes generated by Rab8a Q67L in neurons (Fig. 5H). We co-nucleofected an shRNA against Tuba and Rab8a Q67L in primary cultures of hippocampal neurons and observed that shRNA against Tuba suppress the generation of supernumerary axons induced by Rab8a Q67L and increased the percentage of neurons without axons (Fig. 5I). No changes were observed in the mean minor neurite length, but significant reductions in axonal length in the neurons overexpressing Rab8a Q67L and shRNA against Tuba compared with Rab8a Q67L alone were detected (Fig. 5J,K). Therefore, these results suggest that Tuba can mediate increased Cdc42 activity induced by Rab8a and multiaxonal neurons resulting of Rab8 overexpression could also be dependent on Tuba-triggered activation of Cdc42. 
A

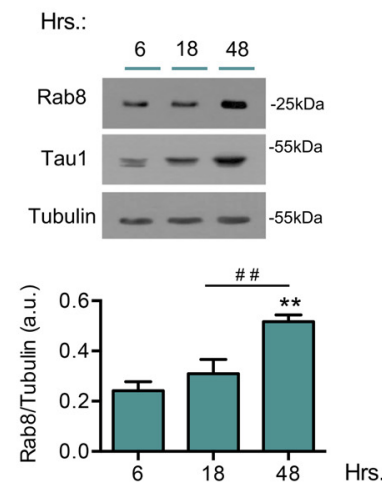

C

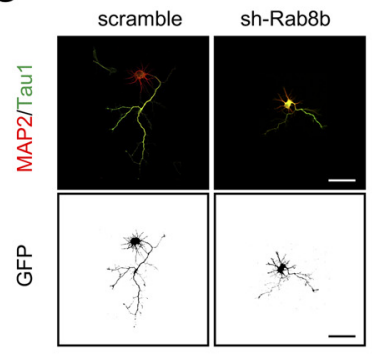

D
B
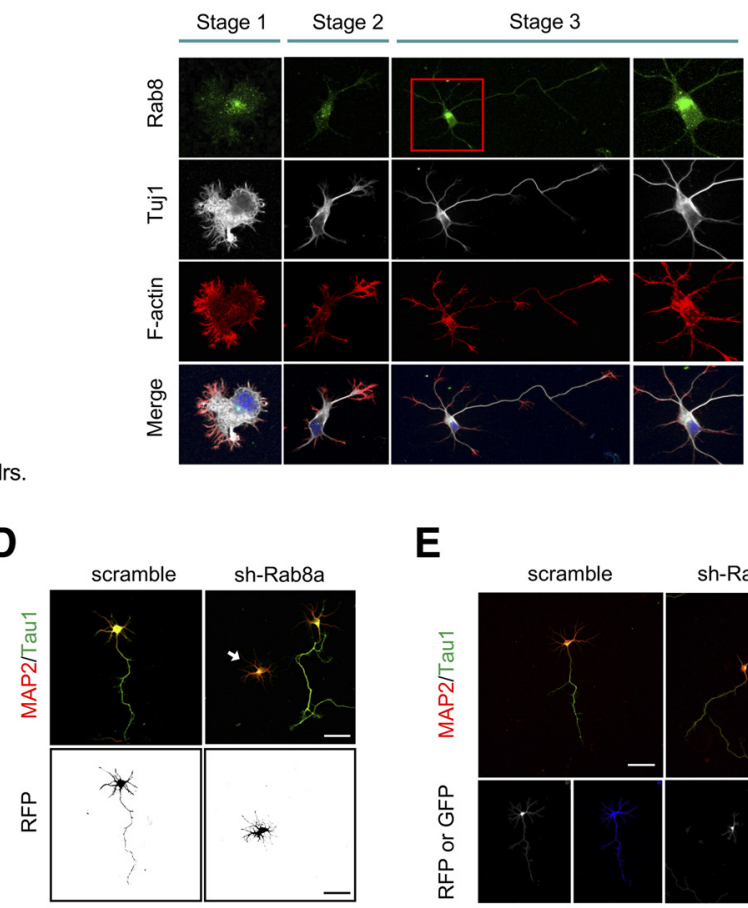

E

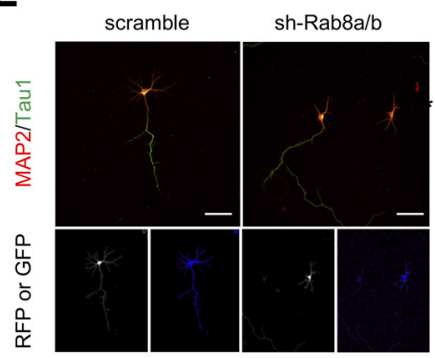

F

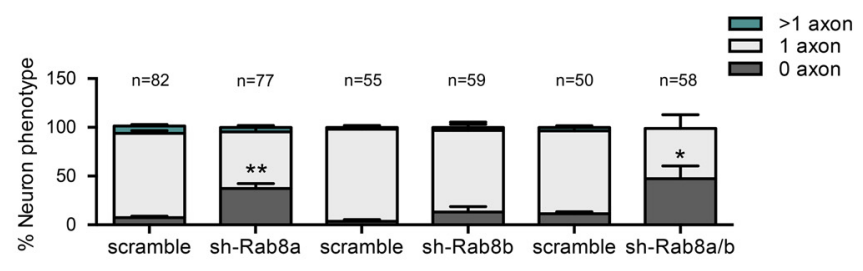

G

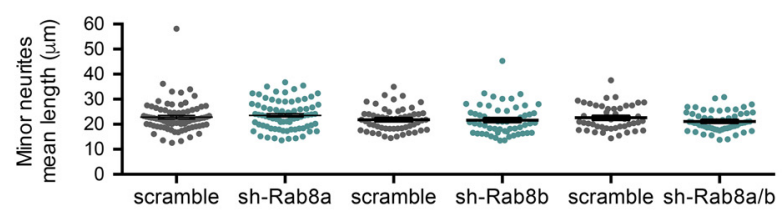

H

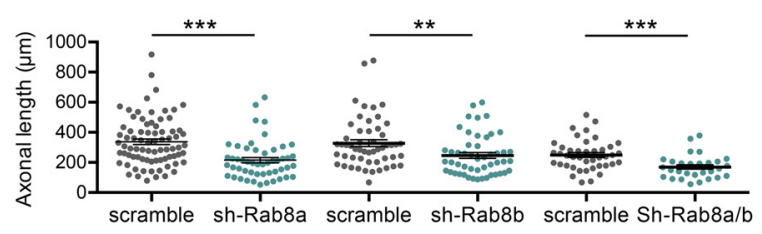

Figure 3. Rab8a is required for axon formation. $\boldsymbol{A}$, Representative Western blotting and quantification of Rab8 expression from cultured cortical neurons at 6,18 , and $48 \mathrm{~h}$ postplating. $\boldsymbol{B}$, Representative images of neurons at stages 1-3 stained with Rab8 (green), Tuj1 (gray), F-actin (red), and T0-PRO-3 (blue). C, Representative images of hippocampal neurons overexpressing scrambled or shRNA against Rab8b, stained with MAP2 and tau-1. D, Representative images of hippocampal neurons overexpressing scrambled or shRNA against Rab8a, stained with MAP2 and tau-1. $\boldsymbol{E}$, Representative images of hippocampal neurons overexpressing scrambled or shRNA against Rab8a and Rab8b (1:1), stained with MAP2 and tau-1. $\boldsymbol{F}$, Quantification of neuron phenotypes of RFP or/and GFP-positive cells in $\boldsymbol{C}-\boldsymbol{E}$. G, Quantification of mean minor neurite length in $\boldsymbol{D}-\boldsymbol{E}$. $\boldsymbol{H}$, Quantification of axonal length in $\boldsymbol{C}-\boldsymbol{E}$. White arrow shows nucleofected neurons. Scale bars: $50 \mu \mathrm{m}$. Values represent mean \pm SEM $(n=3) ; * p<0.05, * * p<0.01, * * * p<0.001$ as compared with the corresponding control; \#\#p $<0.01$ as compared between conditions.

\section{A positive feedback loop between Rab8a and Cdc42 regulates neuronal polarization}

In the next set of experiments, we examined whether the overexpression of a Cdc42 fast cycling mutant (F28L) reversed the loss of polarity induced by overexpression of Rab8a T22N in neurons. We performed co-nucleofection of different forms of Cdc42 and Rab8a and analyzed their effect on neuronal morphology by immunofluorescence against tau-1 and MAP2 (Fig. 6A). We observed that co-expression of Rab8a $\mathrm{T} 22 \mathrm{~N}$ restrain multiaxonal neuronal phenotype induced by 
A

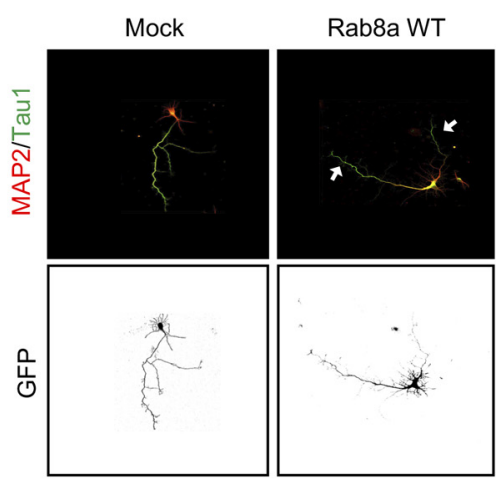

C

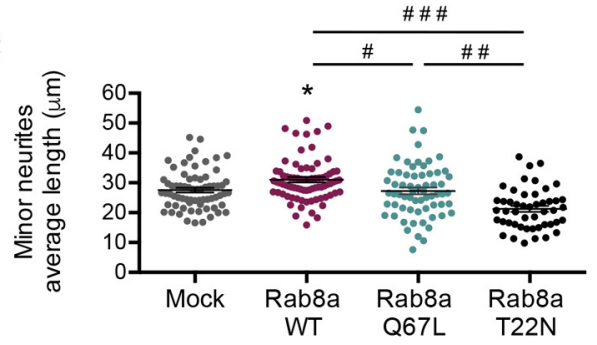

E

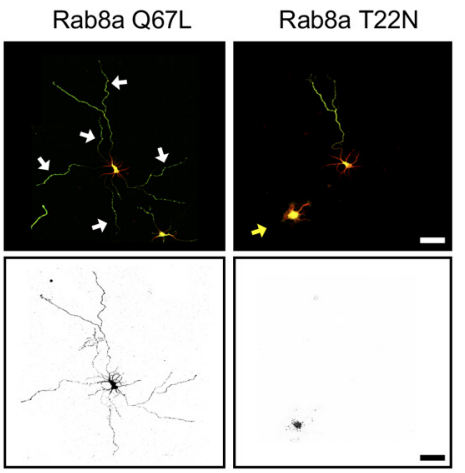

B
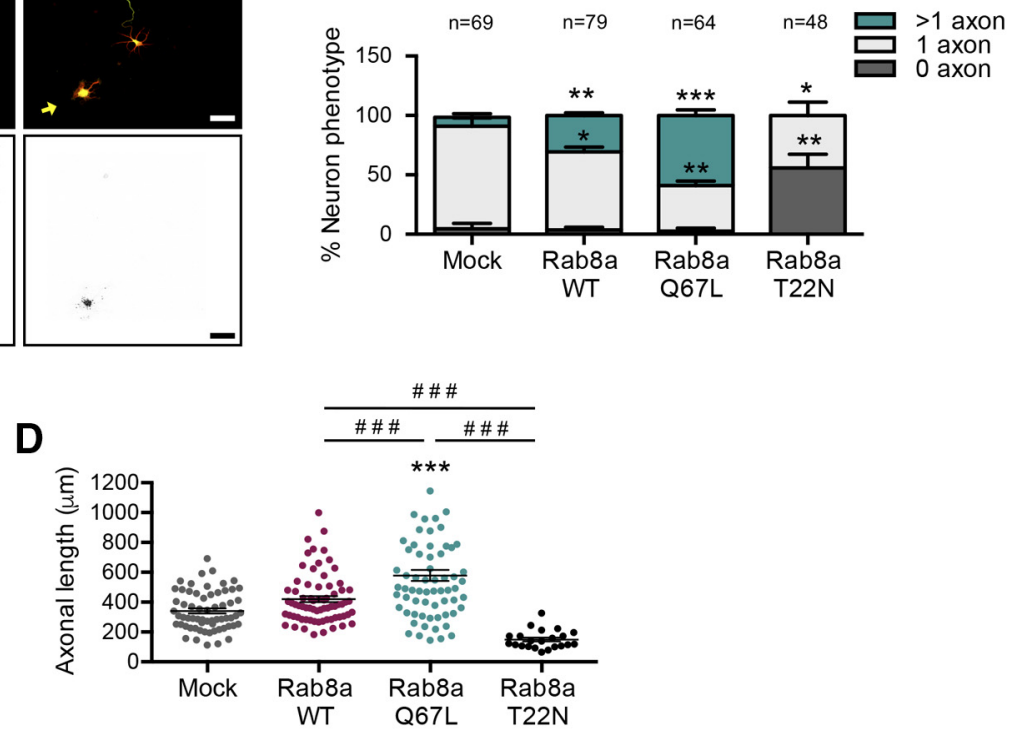

$\mathbf{F}$

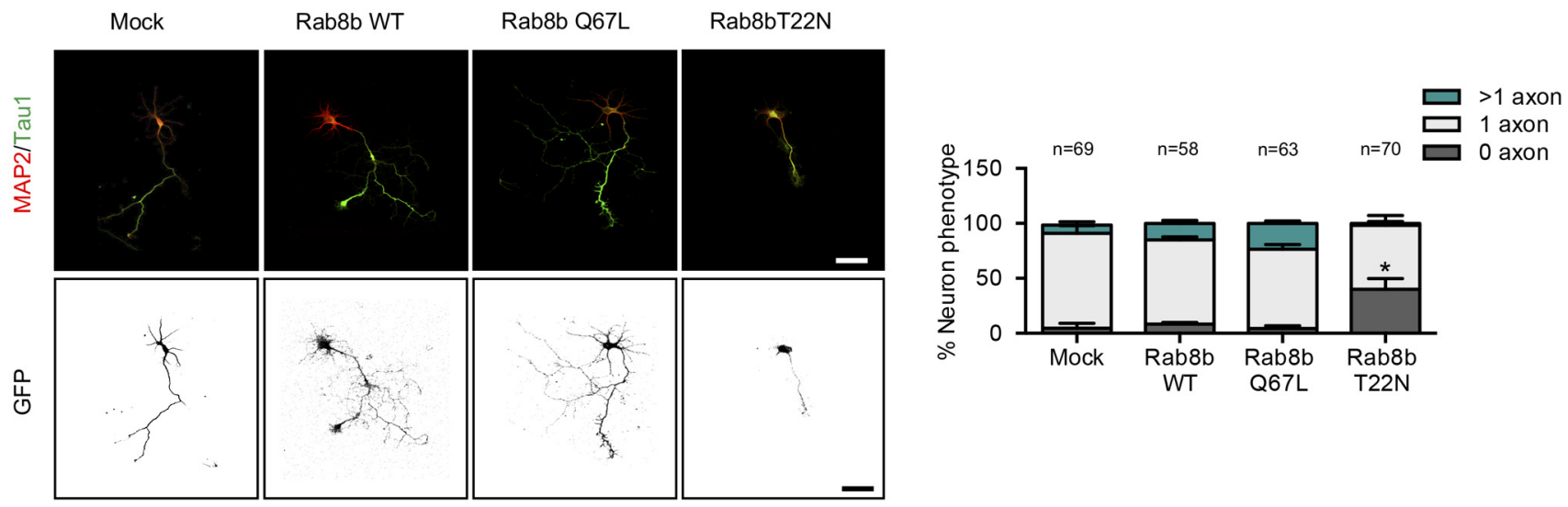

G

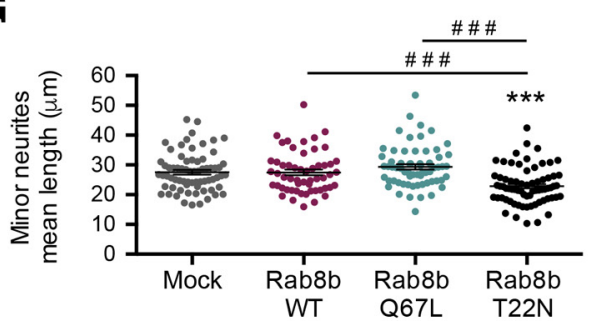

H

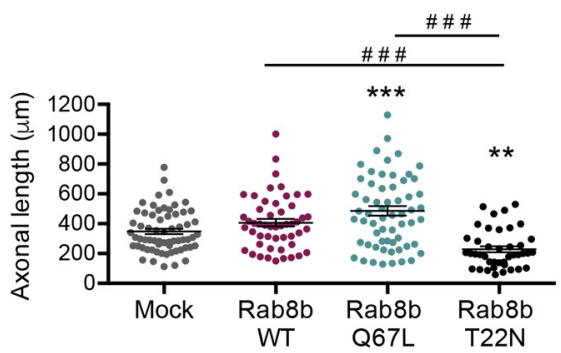

Figure 4. Overexpression of a CA Rab8a isoform generates multiaxonic neurons. $A$, Representative images of hippocampal neurons overexpressing Rab8a WT, Rab8a Q67L (CA mutant), or Rab8a T22N (DN mutant), stained with MAP-2 and tau-1. White arrows indicate axons in the multiaxonic neurons, yellow arrows show nucleofected neurons $B$, Quantification of neuron phenotypes of GFP-positive cells in $\boldsymbol{A}$. C, Quantification of mean minor average length in $\boldsymbol{A}$. D, Quantification of axonal length in $\boldsymbol{A}$. $\boldsymbol{E}$, Representative images of hippocampal neurons overexpressing Rab8b WT, Rab8b Q67L (CA mutant), or Rab8b T22N (DN mutant), stained with MAP2 and tau-1. $\boldsymbol{F}$, Quantification of neuron phenotypes of GFP-positive cells in $\boldsymbol{E}$. G, Quantification of minor neurite average length in $\boldsymbol{E}$. $\boldsymbol{H}$, Quantification of axonal length in $\boldsymbol{E}$. Scale bars: $50 \mu \mathrm{m}$. Values represent mean \pm SEM $(n=3) ; * p<0.05, * * p<0.01$, $* * * p<0.001$ as compared with the corresponding control; \#p <0.05, \#\#p <0.01, \#\#\# <0.001 as compared between conditions. 
A

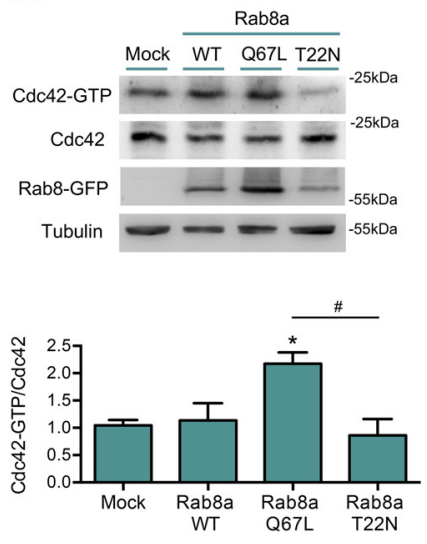

D

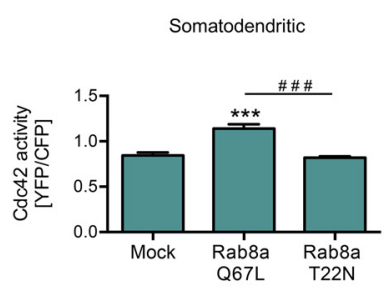

G
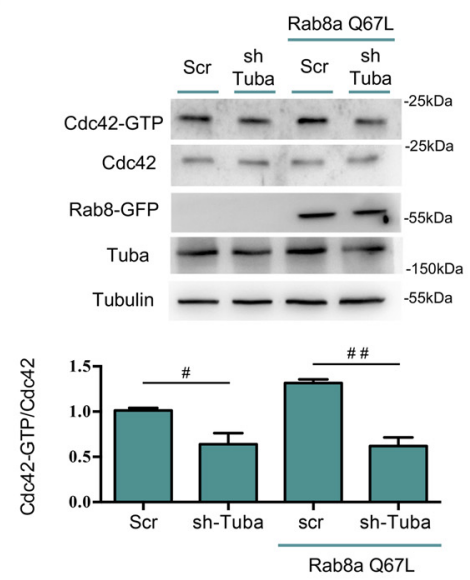

I

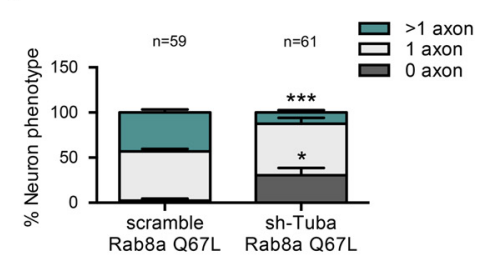

B
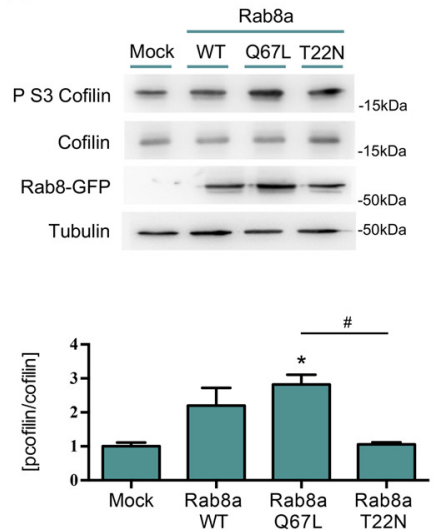

E

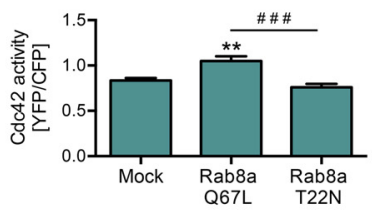

H

\section{H}

C

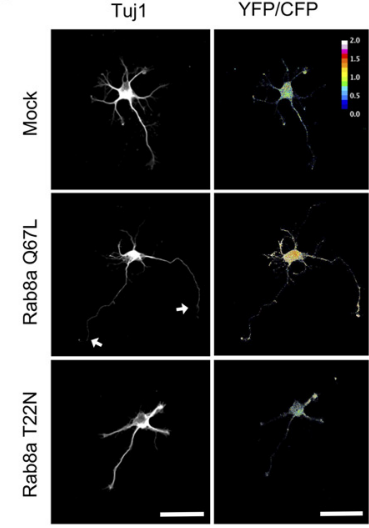

F

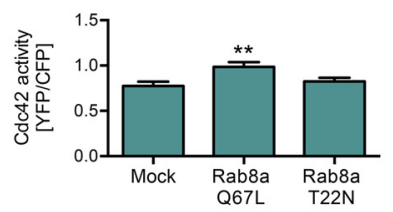

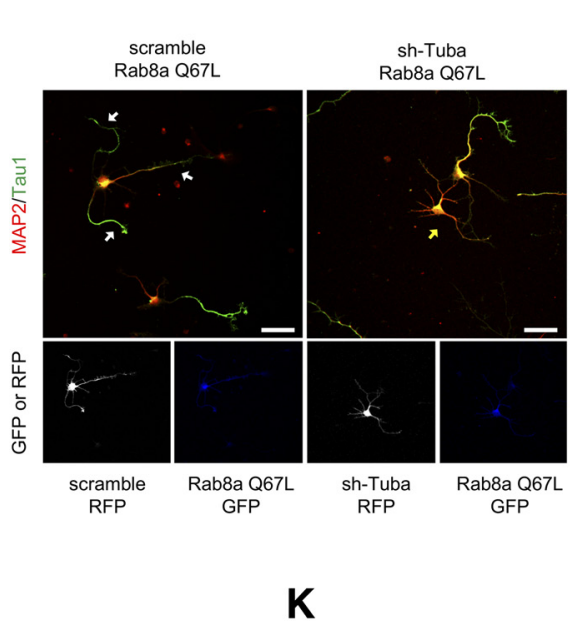
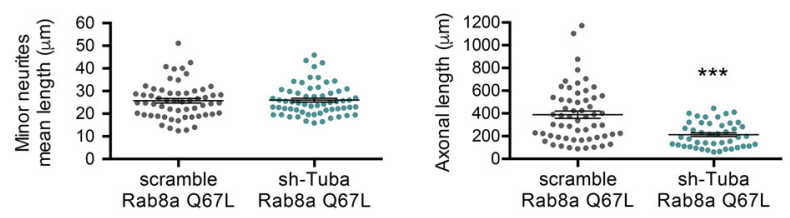

Figure 5. Rab8a CA triggers Tuba-dependent Cdc42 activation. $\boldsymbol{A}$, Representative image and quantification of pulldown assays from N1E-115 neuroblastoma cell lines overexpressing GFP, Rab8a WT, Rab8a Q67L (CA mutant), or Rab8a T22N (DN mutant). B, Representative image and quantification of Western blottings for cofilin and phospho ser3 cofilin from N1E-115 neuroblastoma cell lines overexpressing GFP, Rab8a WT, Rab8a Q67L (CA mutant), or Rab8a T22N (DN mutant). C, One DIV cultured hippocampal neurons expressing a FRET biosensor for Cdc42 coexpressing Flag, Flag-Rab8a Q67L, or Flag-Rab8a T22N and stained against Tuj1 (white). D- $\boldsymbol{F}$, Quantification of the (dc42-GTP/Cdc42 ratio from $\boldsymbol{C}$ in somatodendritic (D), proximal axonal (E), and distal axonal compartments $(\boldsymbol{F})$. G, Representative image and quantification of pulldown assays from N1E-115 neuroblastoma cell lines knock-down for Tuba overexpressing GFP or Rab8a Q67L (CA mutant). $\boldsymbol{H}$, Representative images of Rab8a Q67L-overexpressing neurons co-transfected with a shRNA against Tuba, stained with MAP-2 and tau- 1 . White arrow shows axons in multiaxonic neurons, yellow arrow shows nucleofected neurons. $\boldsymbol{I}$, Quantification of neuron phenotypes of GFP and RFP-positive cells in $\boldsymbol{H}$. $\boldsymbol{J}$, Quantification of minor neurite average length in $\boldsymbol{H}$. $\boldsymbol{K}$, Quantification of axonal length in $\boldsymbol{H}$. Scale bars: $50 \mu \mathrm{m}$. Values represent mean \pm SEM $(n=3) ; * p<0.05, * * p<0.01, * * * p<0.001$ as compared with the corresponding control; $\# p<0.05, \# p<0.01$, \#\#\# <0.001 as compared between conditions.

Cdc42 F28L, while the overexpression of Cdc42 T17N (DN mutant) prevents the generation of supernumerary axons induced by Rab8a Q67L. In addition, the concurrent overexpression of Cdc42 F28L and Rab8a Q67L generated a robust increase in the number of multiaxonal neurons, at levels similar to each one separately (Fig. $6 B$ ). We also stablished that co-expression of Rab8a T22N reduced the minor neurite length of Cdc42 F28L-overexpressing neurons and in turn, 


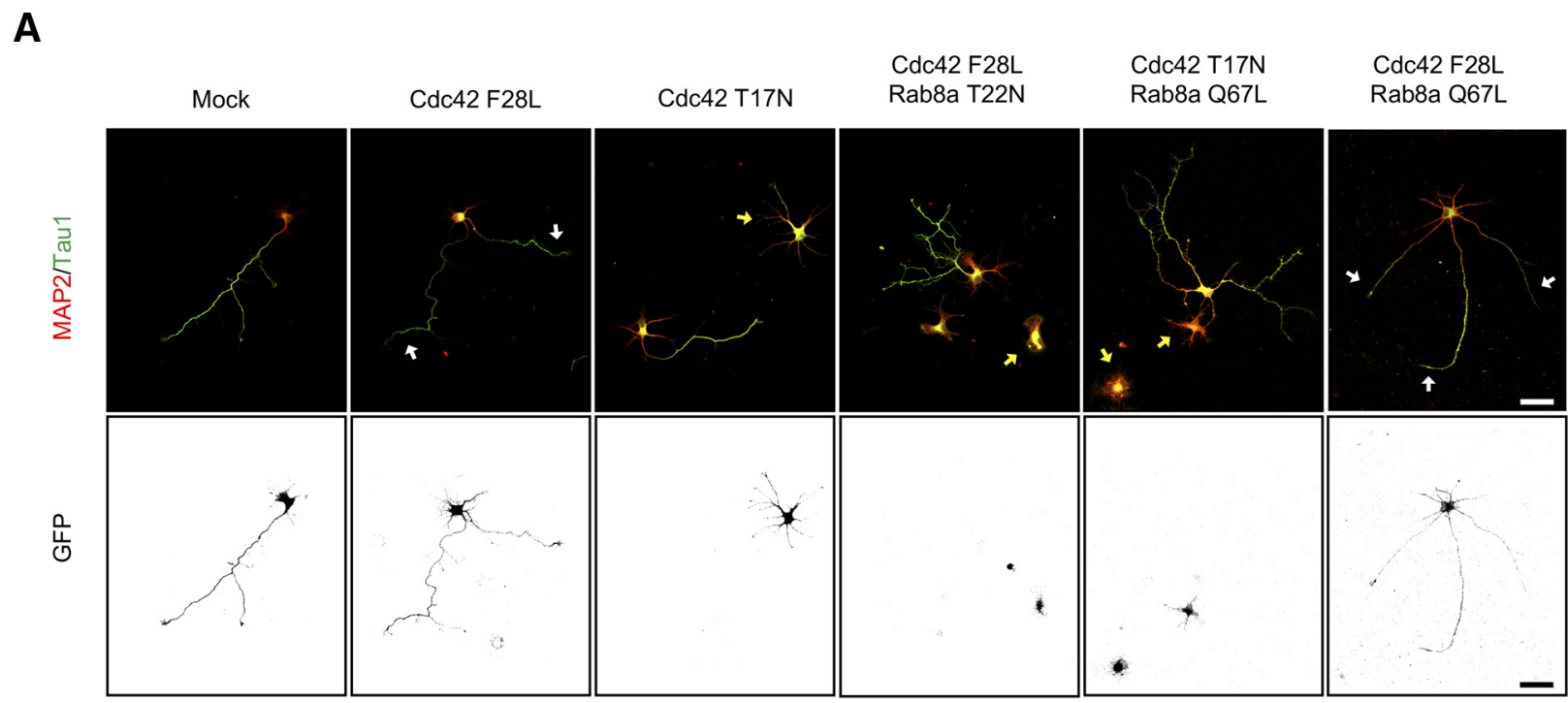

B

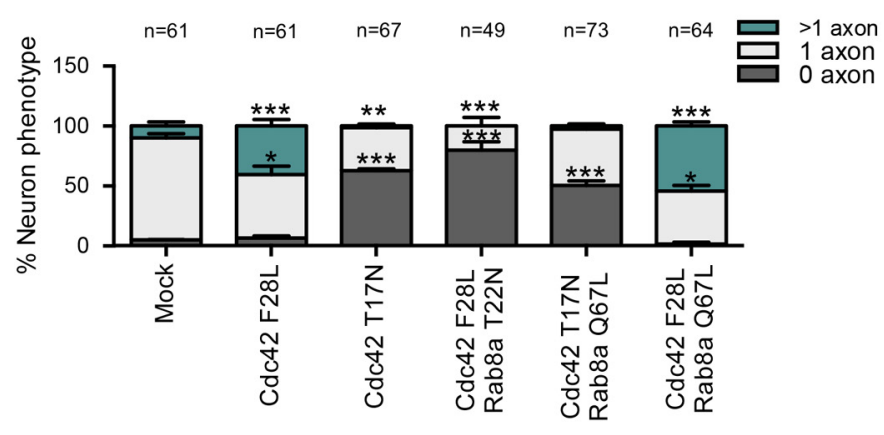

D

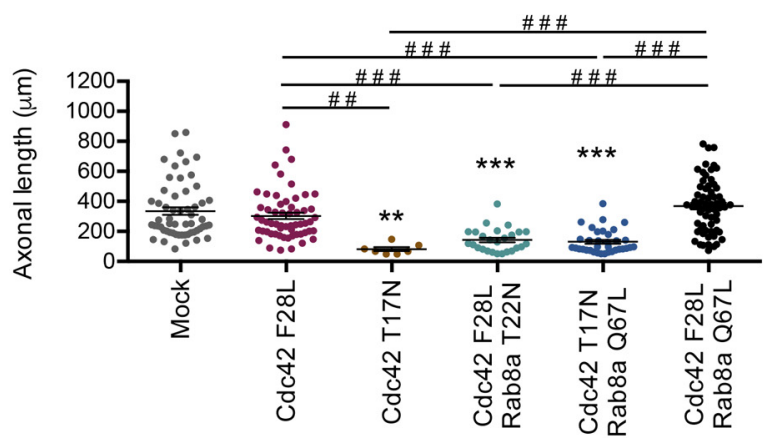

C

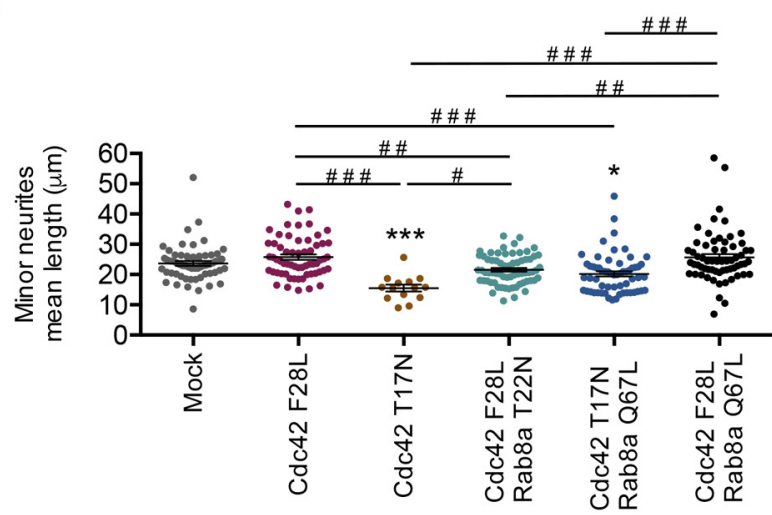

Figure 6. A positive feedback loop between Rab8a and Cdc42 regulates neuronal polarization. $\boldsymbol{A}$, Representative images of Cdc42 F28L (fast cycling mutant) or (dc42 T22N (DN mutant)-0verexpressing neurons co-transfected with Rab8a Q67L or Rab8a T22N, stained with MAP-2 and tau-1. White arrows show axons in multiaxonic neurons, yellow arrows show nucleofected neurons $\boldsymbol{B}$, Quantification of neuron phenotypes of GFP-positive cells in $\boldsymbol{A}$. C, Quantification of minor neurite average length in $\boldsymbol{A}$. $\boldsymbol{D}$, Quantification of axonal length in $\boldsymbol{A}$. Scale bars: $50 \mu \mathrm{m}$. Values represent mean \pm SEM ( $n=3$ ); $* p<0.05, * * p<0.01, * * * p<0.001$ as compared with the corresponding control; \#p $<0.05$, \#\#p $<0.01$, \#\# $<0.001$ as compared between conditions.

the co-expression of Rab8a Q67L increased the minor neurite length of Cdc42 T17N-overexpressing neurons (Fig. 6C). Similarly, co-expression of Rab8a T22N reduced the axonal length of Cdc42 F28L-overexpressing neurons; however, we observed no differences with the co-expression of Rab8a Q67L in Cdc42 T17N-overexpressing neurons (Fig. 6D). These results suggest the existence of a positive feedback loop between Rab8a and Cdc42 that is needed to regulate neuronal polarization.
Tuba and Rab8a regulates the neuronal morphology of migrating neurons at the embryonic cortex

In the developing cortex, axo-dendritic polarity is stablished during neuronal radial migration from $\mathrm{VZ}$ to the $\mathrm{CP}$. Initially, neurons extents multiple minor neurites, and these multipolar cells migrate toward the intermediate zone (IZ) through the subventricular zone (SVZ), changing its morphology to a bipolar one, with a trailing process (future axon) and a leading process (future dendrites). Bipolar cells migrate toward the $\mathrm{CP}$ using radial glia 
A

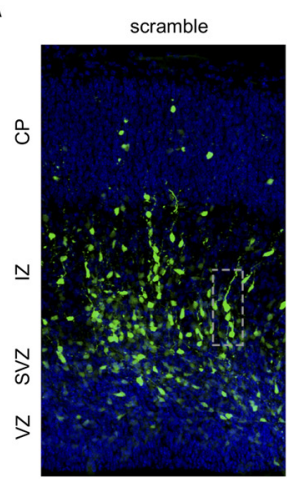

sh-Tuba

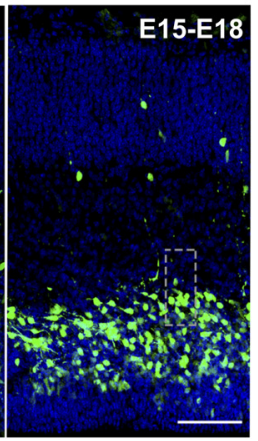

B
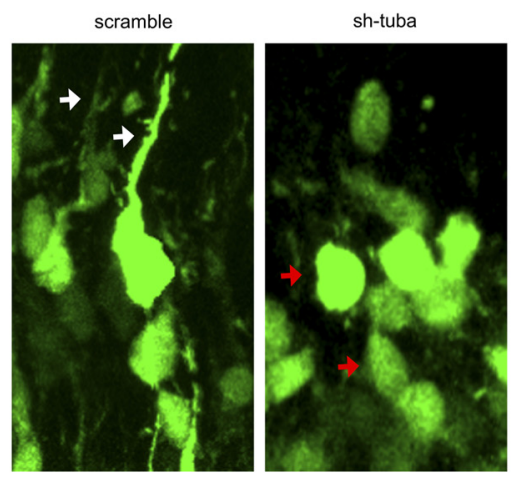

C
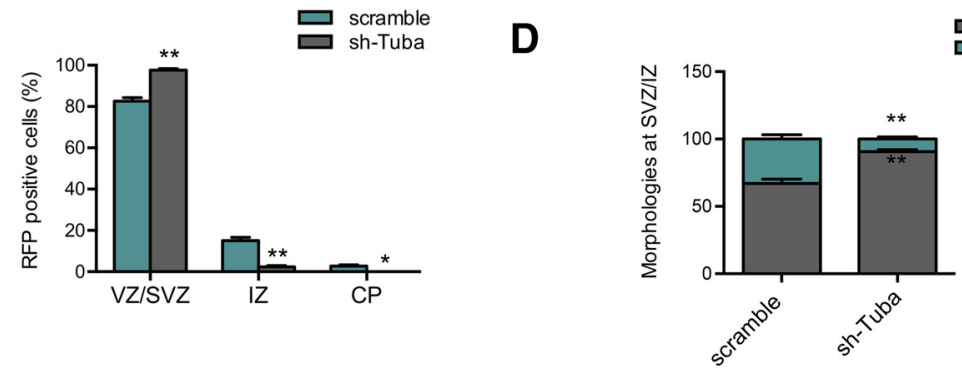
Multipolar

D

E
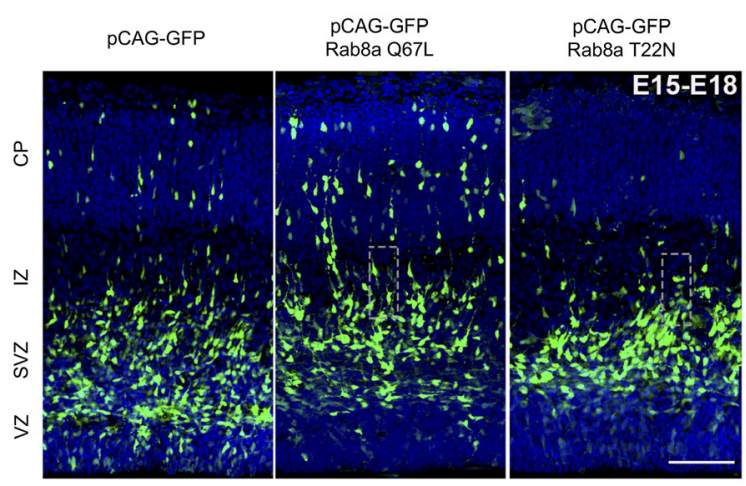

$\mathbf{F}$

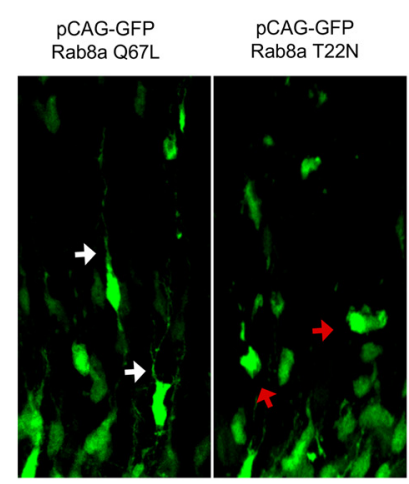

G

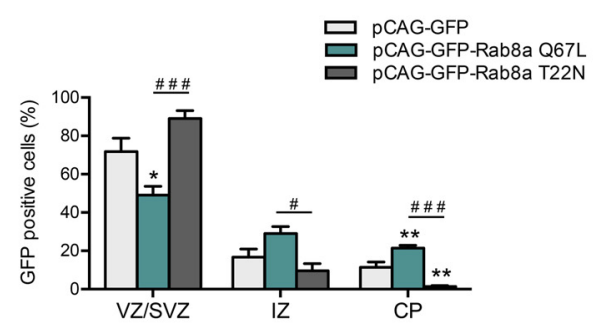

H

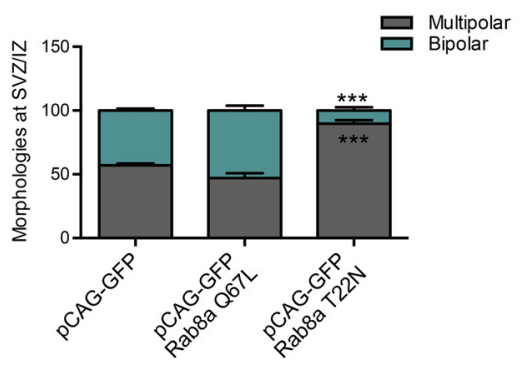

Figure 7. Tuba and Rab8a regulates neuronal morphology of migrating neurons in the embryonic cortex. $A$, Representative images of cortical sections of IUE mice with pRFP-sh-scramble or pRFP-sh-Tuba, at E15 and analyzed at E18. RFP-positive cells (pseudo-colored green) were located in the CP, IZ, SVZ, and VZ. B, Representative image of a zoom between SVZ and IZ of panel $A$. The white arrow shows normal neurons with a bipolar morphology, while the red arrow shows cells with a round morphology. C, Quantification of the distribution of RFP-positive cells in CP, IZ, SVZ, and VZ. D, Quantification of the morphology of RFP-positive cells between IZ and SVZ. E, Representative images of cortical sections of IUE mice with pCAG-GFP, pCAG-GFP plus Rab8a Q67L, or pCAG-GFP plus Rab8a T22N, at E15 and analyzed at E18. $\boldsymbol{F}$, Representative image of a zoom between SVZ and IZ of panel $\boldsymbol{A}$. The white arrow shows normal neurons with a bipolar morphology, while the red arrow shows cells with a round morphology. G, Quantification of the distribution of GFP-positive cells in CP, IZ, SVZ, and VZ. H, Quantification of the morphology of GFP-positive cells between IZ and SVZ. Scale bars: $75 \mu \mathrm{m}$. Values represent mean \pm SEM $(n=4) ; * p<0.05, * * * p<0.01, * * * p<0.001$ as compared with the corresponding control and \#p $<0.05$ and \#\#\# $<0.001$ as compared between conditions.

tracks. The multipolar-to-bipolar transition is a crucial step not only in the establishment of neuronal polarity in vivo (similar to the transition between stages 2 and 3 in cultured neurons) but also in neuronal migration in developing cortex (Takano et al.,
2019). Therefore, analysis of the morphology and migration of IUE neuronal precursors, constitute the gold standard for evaluate molecular determinants of neuronal polarity in vivo. To this end, we performed IUE of E15 embryos with plasmids encoding 
shRNA against Tuba or a scrambled control into the lateral ventricle, followed by fixation at E18. We analyzed migration of neurons in 4 different cortical layers: VZ, SVZ, IZ, and CP (Fuentes et al., 2012). Scrambled electroporated neurons (visualized through RFP, pseudo-colored green) showed normal migration patterns and morphology. In contrast, the number of Tuba knock-down neurons that reached the IZ and CP was significantly reduced, accumulating in the lower cortical layers VZSVZ (Fig. 7A,C). At individual level, cortical neurons underwent a multipolar-to-bipolar transition in the SVZ-IZ to migrate toward the CP (Yogev and Shen, 2017). Therefore, we analyzed the prevalence of multipolar and bipolar RFP-positive neurons in the SVZ-IZ in sh-scramble or sh-Tuba electroporated animals (Fig. 7A-D). We found that sh-scramble expressing neurons, $67.04 \pm 3.24 \%$ have a multipolar morphology and $32.96 \pm 3.24 \%$ changed to a bipolar morphology (Fig. $7 B$, white arrow). In contrast, $90.65 \pm 1.51 \%$ of the Tuba knock-down neurons remain arrested in a multipolar state. Moreover, several Tuba knockdown neurons displayed a round morphology (Fig. 7B, red arrow)

Rab8a knock-down had previously been reported to impairs cortical neuronal differentiation in vivo (Wang et al., 2011). To complement these findings, E15 embryos were electroporated to express pCAG-GFP vector with the cDNAs of Rab8a T22N or Rab8a Q67L into the lateral ventricle, followed by fixation at E18. Control electroporated neurons (visualized through eGFP) showed normal migration patterns. Interestingly, the number of neurons expressing Rab8a Q67L that reached the CP was significantly higher whereas that number of neurons expressing Rab8a $\mathrm{T} 22 \mathrm{~N}$ that reached the $\mathrm{CP}$ was significantly reduced, compared with control neurons (Fig. $7 E, G$ ). In addition, we observed a reduction in the number of neurons expressing Rab8a Q67L that remained at VZ and SVZ regions (Fig. 7E,G).

We also analyzed the presence and prevalence of multipolar and bipolar Rab8a T22N GFP-positive or Rab8a Q67L GFP-positive neurons in the SVZ-IZ area compared with control (Fig. $7 E$, $F, H)$. We found that control and Rab8a Q67L-expressing neurons display similar proportions of multipolar and bipolar morphologies. In contrast, $89.74 \pm 2.63 \%$ of Rab8a T22N-expressing neurons have a multipolar morphology, with a few round cells (Fig. 7F, red arrow). Concomitantly, the proportion of bipolar neurons, that correspond to proper polarized migrating neurons, was significantly reduced. Altogether, our results indicate that Tuba and Rab8a regulates neuronal polarity acquisition and migration in vivo, reinforcing the notion that both play a critical role instructing axon determination and outgrowth.

\section{Discussion}

\section{Role of tuba in neuronal polarity acquisition}

In this report, we showed that two small GTPases, Rab8 and Cdc42, are crucial molecular determinants for axon specification and that they are molecularly linked by Tuba.

Tuba is a multidomain scaffolding protein that consists of four N-terminal SH3 domains that bind directly to dynamin1, an internal Bin, amphiphysin, Rvs (BAR) domain, a GEF domain specific for the Rho-family GTPase Cdc42 (generating an intermediate activation) and two C-terminal SH3 domains. The extreme C-terminal SH3 domain directly binds to multiple actinregulatory proteins, including N-WASP and Ena/VASP (Salazar et al., 2003; Kovacs et al., 2006). There are two isoforms of Tuba generated by alternative splicing, that differ by the presence of $\mathrm{N}$ terminal SH3 domains, named TubaFL $(\sim 180 \mathrm{kDa})$ and mTuba $(\sim 75 \mathrm{kDa})$. Tuba have a well stablished role in epithelial cell polarization. In Madin-Darby canine kidney (MDCK) cysts, Tuba silencing causes defective lumen formation, randomization of mitotic spindle orientation and reduced aPKC activity. Tuba is located below the apical cortex and is essential for Cdc42 enrichment at this site (Qin et al., 2010). On the same line, Par6a, Cdc42, and Tuba form a trimeric complex and colocalize in the apical domain of polarized enterocytes (Bruurs et al., 2018), where Tuba is responsible for immobilizing active Cdc42 to ensure its enrichment at apical plasma membrane (Bruurs et al., 2017). In epithelial polarity, Tuba is integrated in a complex molecular network, downstream of Rab11a-Rabin8-Rab8a cascade (Bryant et al., 2010) and upstream of Cdc42 and its effectors that includes the Par polarity complex and the actin regulator $\mathrm{N}$ WASP (Otani et al., 2006; Kovacs et al., 2011). However, its role in neuronal polarity has not been addressed previously.

Similarly to epithelial cells, in developing neurites, activation of the Par6-Par3-aPKC-Cdc42 cascade is required for axon growth (Lalli, 2009), but the identity of the GEF involved in Cdc42 activation had not been described until now. Our results indicate that Tuba connects Rab8 and Cdc42 activities which are essential to define axon identity. Tuba enrichment at the axonal growth cone tip recapitulates the behavior of other proteins involved in neuronal polarity. For example, before axon specification, Par3 is localized in the cell body and at the tips of all nascent processes (Shi et al., 2003; Schwamborn and Püschel, 2004; Nishimura et al., 2005), whereas in stage $3, \operatorname{Par} 3$ is lost from minor neurites and becomes selectively accumulated in the axon and developing growth cone (Shi et al., 2003). The spatial and temporal distribution of Par6 during axon specification is similar to Par3, being to the cell body and the axon at stage 3 (Shi et al., 2003; Schwamborn and Püschel, 2004).

The relevance of Tuba in axon specification is reinforced by loss-of-function and gain-of-function approaches that highlight antagonistic effects over neuronal polarity acquisition. Consistently, loss-of-function and gain-of-function for Cdc42 lead to similar results (Schwamborn and Püschel, 2004; Konno et al., 2005; Garvalov et al., 2007; Govek et al., 2018; López Tobón et al., 2018). In addition, a truncated isoform of Tuba called mTuba had no effect on neuronal polarity. Unlike mTuba, TubaFL can bind dynamin (Kovacs et al., 2006) and GM130, controlling Cdc42 activation at the Golgi apparatus (Kodani et al., 2009). Cdc42 was the first Rho family protein to be localized to the Golgi (Erickson et al., 1996) and numerous evidence indicates that Golgi-associated Cdc42 plays a central role in cell polarity. GM130 silencing reduces both total and Golgi-associated Cdc42-GTP, impairing polarization of migrating and epithelial cells. Interestingly, blockade of vesicle trafficking from Golgi reduces Cdc42-GTP accumulation in the leading edge of migrating cells (Baschieri et al., 2014). Tuba plays an essential role in Cdc42 activation in the Golgi apparatus, since Tuba knock-down reduces both Golgi and plasma membrane Cdc42 activity (Herrington et al., 2017). Moreover, another Golgi-associated GEF, named TRIO, has been shown to be important for neuritic outgrowth in cerebellar granule neurons in a Rab8/10-dependent mechanism (Tao et al., 2019), pinpointing the relevance of postGolgi trafficking in neuronal polarity. Therefore, the results presented here showed that Tuba has an essential role in the acquisition of neuronal polarity in vitro and in vivo, which suggests that neurons and epithelial cells not only share their embryonic origin, but also the mechanisms that allow them to adopt their morphologic asymmetry. 


\section{The small GTPase Rab8, a relevant factor in neuronal polarity}

A conceptual framework proposed that the development of neuronal polarity encompasses three phases: demarcation, growth, and commitment. The first involves the generation of the first neurite, followed by axon specification and elongation; and ending with the third phase with the functional/physiological differentiation between the axon and dendrites. Each phase must be preceded by a spatial restriction for growth activity (Cáceres et al., 2012). Therefore, growth processes are intricately linked to the development of neuronal polarity. The secretory pathway is the primary source for newly synthesized membranes and is essential for axon spreading and growth. Rab8 was originally described as a trafficking regulator between the TGN and the plasma membrane and its overexpression induce the formation of long cell surface protrusions in a variety of cells (Huber et al., 1995; Peränen et al., 1996; Hattula and Peränen, 2000). A role for Rab8 in neurite elongation was proposed earlier (Huber et al., 1995); however its role as an axon determinant is novel. There are two Rab8 isoforms, Rab8a and Rab8b, which are encoded by two different genes. A recent report using high throughput screening shows that both isoforms are present in neurons, although Rab8a is expressed at higher levels (Sharma et al., 2015). We analyzed the effect of both Rab8 isoforms on neuronal polarity and concluded that only neurons overexpressing Rab8a CA have supernumerary axons, whereas neurons overexpressing Rab8a DN or Rab8b DN have a greater number of neurons arrested in stage 2, without axon. In contrast, a previous study in primary mouse cortical neurons reports that overexpression of Rab8a CA generates longer axons, whereas neurons overexpressing Rab8a DN does not have differences in axonal length compared with control neurons. Those authors suggested a functional coupling between Rab8 and Rab11, likely at the recycling endosomes (Furusawa et al., 2017). A role for Rab8 in axon specification is also supported by in vivo experiments showing that reducing Rab8a activity in the brain significantly increases the proportion of multipolar neurons in the cortex. Similar phenotypes were found by targeting other molecular components involved in neuronal polarity acquisition such as IGF-1R (Nieto Guil et al., 2017) and Par3 (Funahashi et al., 2013).

Several lines of evidence suggest that key processes determining neuronal morphology, such as axon specification, elongation and branching could be dissected at a molecular level, based on their dependence on Rab-specific vesicular trafficking regulation (Villarroel-Campos et al., 2014, 2016a). The early/late endosome Rab GTPases: Rab5, Rab7, Rab21, and Rab22 have been mainly associated with the regulation of neurite outgrowth. The recycling endosome-associated Rab GTPases: Rab4, Rab11, Rab35 have been linked with the regulation of axonal elongation. Finally, TGN-related Rab GTPases, as Rab10 and Rab33, have been reported to regulate axonal elongation and axonal specification, respectively (Villarroel-Campos et al., 2014, 2016a). The axon determination during neuronal polarization is preceded by bulk cytoplasmic flow and polarized TGN-derived vesicle transport toward one specific neurite (Bradke and Dotti, 1997; Calderon de Anda et al., 2008). Thus, Rabs involved in traffic regulation from the Golgi apparatus could be relevant in axonal specification. Therefore, we envision the existence of two pools of Rab8, one in the recycling pathway that regulates axonal elongation, and a second pool that controls exocytic traffic from the TGN regulating axonal specification.
We also found a connection between Rab8a and actin dynamics. We observed that Rab8 activation induces a Tubamediated increase in Cdc42 activity, and that the effect of Rab8a overexpression on neuron morphology is dependent on Tuba expression.

\section{A positive feedback loop between Rab8a and Cdc42 regulates neuronal polarization}

Positive feedback circuits are crucial during neuronal polarization, contributing to stochastic selection of one of the minor neurites to become the axon and simultaneously preventing the axonal identity of the other minor neurites. Examples of those positive feedback loops during neuronal polarity acquisition are observed during the coupling of Ras, PI3K and Cdc42/ Par3/Par6/Rac1 signaling pathways (Barnes and Polleux, 2009). We observed that Cdc42 fast cycling overexpression cannot overcome the phenotype induced in the Rab8a DN mutant. Concomitantly, Rab8a CA overexpression cannot reverse the phenotype induced by the Cdc42 DN mutant, suggesting a reciprocal regulation. Previous reports showed that $\mathrm{Cdc} 42$ regulates Golgi organization and post-Golgi traffic (Friesland et al., 2013; Kage et al., 2017) emphasizing the importance of post-Golgi traffic and therefore a putative involvement of Rab8 in this process. Additionally in another highly polarized tissue, the mouse intestine, Cdc42 deficiency impaired both Rab8a vesicle trafficking to the midbody during cytokinesis and Rab8a activation and its subsequent association with effectors (Sakamori et al., 2012).

In conclusion, the molecular mechanism described here located Tuba as the Cdc42 GEF involved in axon specification and suggest that Tuba connects cytoskeleton dynamics and the directed vesicular traffic during neuronal polarization.

\section{References}

Arimura N, Kaibuchi K (2007) Neuronal polarity: from extracellular signals to intracellular mechanisms. Nat Rev Neurosci 8:194-205.

Armstrong J, Thompson N, Squire JH, Smith J, Hayes B, Solari R (1996) Identification of a novel member of the Rab8 family from the rat basophilic leukaemia cell line, RBL.2H3. J Cell Sci 109:1265-1274.

Barnes AP, Polleux F (2009) Establishment of axon-dendrite polarity in developing neurons. Annu Rev Neurosci 32:347-381.

Baschieri F, Confalonieri S, Bertalot G, Di Fiore PP, Dietmaier W, Leist M, Crespo P, Macara IG, Farhan H (2014) Spatial control of Cdc42 signalling by a GM130-RasGRF complex regulates polarity and tumorigenesis. Nat Commun 5:4839.

Bonifacino JS (2014) Adaptor proteins involved in polarized sorting. J Cell Biol 204:7-17.

Bradke F, Dotti CG (1997) Neuronal polarity: vectorial cytoplasmic flow precedes axon formation. Neuron 19:1175-1186.

Bradke F, Dotti CG (2000) Establishment of neuronal polarity: lessons from cultured hippocampal neurons. Curr Opin Neurobiol 10:574-581.

Bruurs LJM, Zwakenberg S, van der Net MC, Zwartkruis FJ, Bos JL (2017) A two-tiered mechanism enables localized Cdc42 signaling during enterocyte polarization. Mol Cell Biol 37:e00547-16.

Bruurs LJM, van der Net MC, Zwakenberg S, Zwartkruis FJT, Bos JL (2018) A Tuba/Cdc42/Par6A complex is required to ensure singularity in apical domain formation during enterocyte polarization. PLoS One 13: e0207159.

Bryant DM, Datta A, Rodríguez-Fraticelli AE, Peränen J, Martín-Belmonte F, Mostov KE (2010) A molecular network for de novo generation of the apical surface and lumen. Nat Cell Biol 12:1035-1045.

Cáceres A, Ye B, Dotti CG (2012) Neuronal polarity: demarcation, growth and commitment. Curr Opin Cell Biol 24:547-553.

Calderon de Anda F, Gärtner A, Tsai LH, Dotti CG (2008) Pyramidal neuron polarity axis is defined at the bipolar stage. J Cell Sci 121:178-185. 
Casoli T, Di Stefano G, Fattoretti P, Giorgetti B, Balietti M, Lattanzio F, Aicardi G, Platano D (2012) Dynamin binding protein gene expression and memory performance in aged rats. Neurobiol Aging 33:618.e15-9.

Cestra G, Kwiatkowski A, Salazar M, Gertler F, De Camilli P (2005) Tuba, a GEF for CDC42, links dynamin to actin regulatory proteins. Methods Enzymol 404:537-545.

Cheng PL, Poo MM (2012) Early events in axon/dendrite polarization. Annu Rev Neurosci 35:181-201.

Dotti CG, Sullivan CA, Banker GA (1988) The establishment of polarity by hippocampal neurons in culture. J Neurosci 8:1454-1468.

Erickson JW, Zhang C, Kahn RA, Evans T, Cerione RA (1996) Mammalian Cdc42 is a brefeldin A-sensitive component of the Golgi apparatus. J Biol Chem 271:26850-26854.

Friesland A, Zhao Y, Chen YH, Wang L, Zhou H, Lu Q (2013) Small molecule targeting Cdc42-intersectin interaction disrupts Golgi organization and suppresses cell motility. Proc Natl Acad Sci USA 110:1261-1266.

Fuentes P, Cánovas J, Berndt FA, Noctor SC, Kukuljan M (2012) CoREST/ LSD1 control the development of pyramidal cortical neurons. Cereb Cortex 22:1431-1441.

Fukuda M (2003) Distinct Rab binding specificity of Rim1, Rim2, rabphilin, and Noc2. Identification of a critical determinant of Rab3A/Rab27A recognition by Rim2. J Biol Chem 278:15373-15380.

Funahashi Y, Namba T, Fujisue S, Itoh N, Nakamuta S, Kato K, Shimada A, Xu C, Shan W, Nishioka T, Kaibuchi K (2013) ERK2-mediated phosphorylation of Par3 regulates neuronal polarization. J Neurosci 33:1327013285.

Furusawa K, Asada A, Urrutia P, Gonzalez-Billault C, Fukuda M, Hisanaga SI (2017) Cdk5 regulation of the GRAB-mediated Rab8-Rab11 cascade in axon outgrowth. J Neurosci 37:790-806.

Garvalov BK, Flynn KC, Neukirchen D, Meyn L, Teusch N, Wu X, Brakebusch C, Bamburg JR, Bradke F (2007) Cdc42 regulates cofilin during the establishment of neuronal polarity. J Neurosci 27:13117-13129.

Govek EE, Wu Z, Acehan D, Molina H, Rivera K, Zhu X, Fang Y, TessierLavigne M, Hatten ME (2018) Cdc42 regulates neuronal polarity during cerebellar axon formation and glial-guided migration. iScience 1:35-48.

Hattula K, Peränen J (2000) FIP-2, a coiled-coil protein, links Huntingtin to Rab8 and modulates cellular morphogenesis. Curr Biol 10:1603-1606.

Herrington KA, Trinh AL, Dang C, O'Shaughnessy E, Hahn KM, Gratton E, Digman MA, Sütterlin C (2017) Spatial analysis of Cdc42 activity reveals a role for plasma membrane-associated $\mathrm{Cdc} 42$ in centrosome regulation. Mol Biol Cell 28:2135-2145.

Homma Y, Fukuda M (2016) Rabin8 regulates neurite outgrowth in both GEF activity-dependent and -independent manners. Mol Biol Cell 27:2107-2118.

Huber LA, Dupree P, Dotti CG (1995) A deficiency of the small GTPase rab8 inhibits membrane traffic in developing neurons. Mol Cell Biol 15:918924

Kaech S, Banker G (2006) Culturing hippocampal neurons. Nat Protoc 1:2406-2415.

Kage F, Steffen A, Ellinger A, Ranftler C, Gehre C, Brakebusch C, Pavelka M, Stradal T, Rottner K (2017) FMNL2 and -3 regulate Golgi architecture and anterograde transport downstream of Cdc42. Sci Rep 7:9791.

Kodani A, Kristensen I, Huang L, Sütterlin C (2009) GM130-dependent control of Cdc42 activity at the Golgi regulates centrosome organization. Mol Biol Cell 20:1192-1200.

Konno D, Yoshimura S, Hori K, Maruoka H, Sobue K (2005) Involvement of the phosphatidylinositol 3-kinase/racl and cdc42 pathways in radial migration of cortical neurons. J Biol Chem 280:5082-5088.

Kovacs EM, Makar RS, Gertler FB (2006) Tuba stimulates intracellular NWASP-dependent actin assembly. J Cell Sci 119:2715-2726.

Kovacs EM, Verma S, Thomas SG, Yap AS (2011) Tuba and N-WASP function cooperatively to position the central lumen during epithelial cyst morphogenesis. Cell Adh Migr 5:344-350.

Lalli G (2009) RalA and the exocyst complex influence neuronal polarity through PAR-3 and aPKC. J Cell Sci 122:1499-1506.

Lalli G (2014) Regulation of neuronal polarity. Exp Cell Res 328:267-275.

López Tobón A, Suresh M, Jin J, Vitriolo A, Pietralla T, Tedford K, Bossenz M, Mahnken K, Kiefer F, Testa G, Fischer KD, Püschel AW (2018) The guanine nucleotide exchange factor Arhgef7/betaPix promotes axon formation upstream of TC10. Sci Rep 8:8811.

Mestres I, Chuang JZ, Calegari F, Conde C, Sung CH (2016) SARA regulates neuronal migration during neocortical development through L1 trafficking. Development 143:3143-3153.

Nakamura T, Kurokawa K, Kiyokawa E, Matsuda M (2006) Analysis of the spatiotemporal activation of rho GTPases using Raichu probes. Methods Enzymol 406:315-332.

Nieto Guil AF, Oksdath M, Weiss LA, Grassi DJ, Sosa LJ, Nieto M, Quiroga S (2017) IGF-1 receptor regulates dynamic changes in neuronal polarity during cerebral cortical migration. Sci Rep 7:7703.

Nishimura T, Yamaguchi T, Kato K, Yoshizawa M, Nabeshima Y, Ohno S, Hoshino M, Kaibuchi K (2005) PAR-6-PAR-3 mediates Cdc42-induced Rac activation through the Rac GEFs STEF/Tiam1. Nat Cell Biol 7:270277.

Otani T, Ichii T, Aono S, Takeichi M (2006) Cdc42 GEF Tuba regulates the junctional configuration of simple epithelial cells. J Cell Biol 175:135146.

Peränen J, Auvinen P, Virta H, Wepf R, Simons K (1996) Rab8 promotes polarized membrane transport through reorganization of actin and microtubules in fibroblasts. J Cell Biol 135:153-167.

Pichaud F, Walther RF, Nunes de Almeida F (2019) Regulation of Cdc42 and its effectors in epithelial morphogenesis. J Cell Sci 132:jcs217869.

Qin Y, Meisen WH, Hao Y, Macara IG (2010) Tuba, a Cdc42 GEF, is required for polarized spindle orientation during epithelial cyst formation. J Cell Biol 189:661-669.

Sakamori R, Das S, Yu S, Feng S, Stypulkowski E, Guan Y, Douard V, Tang W, Ferraris RP, Harada A, Brakebusch C, Guo W, Gao N (2012) Cdc42 and Rab8a are critical for intestinal stem cell division, survival, and differentiation in mice. J Clin Invest 122:1052-1065.

Salazar MA, Kwiatkowski AV, Pellegrini L, Cestra G, Butler MH, Rossman KL, Serna DM, Sondek J, Gertler FB, De Camilli P (2003) Tuba, a novel protein containing bin/amphiphysin/Rvs and Dbl homology domains, links dynamin to regulation of the actin cytoskeleton. J Biol Chem 278:49031-49043.

Schwamborn JC, Püschel AW (2004) The sequential activity of the GTPases Rap1B and Cdc42 determines neuronal polarity. Nat Neurosci 7:923929.

Sharma K, Schmitt S, Bergner CG, Tyanova S, Kannaiyan N, ManriqueHoyos N, Kongi K, Cantuti L, Hanisch UK, Philips MA, Rossner MJ, Mann M, Simons M (2015) Cell type- and brain region-resolved mouse brain proteome. Nat Neurosci 18:1819-1831.

Shi SH, Jan LY, Jan YN (2003) Hippocampal neuronal polarity specified by spatially localized $\mathrm{mPar} 3 / \mathrm{mPar}$ and PI 3-kinase activity. Cell 112:63-75.

Stenmark H (2009) Rab GTPases as coordinators of vesicle traffic. Nat Rev Mol Cell Biol 10:513-525.

Takano T, Funahashi Y, Kaibuchi K (2019) Neuronal polarity: positive and negative feedback signals. Front Cell Dev Biol 7:69.

Tao T, Sun J, Peng Y, Li Y, Wang P, Chen X, Zhao W, Zheng YY, Wei L, Wang W, Zhou Y, Liu J, Shi YS, Zhu MS (2019) Golgi-resident TRIO regulates membrane trafficking during neurite outgrowth. J Biol Chem 294:10954-10968

Villarroel-Campos D, Gastaldi L, Conde C, Caceres A, Gonzalez-Billault C (2014) Rab-mediated trafficking role in neurite formation. J Neurochem 129:240-248.

Villarroel-Campos D, Bronfman FC, Gonzalez-Billault C (2016a) Rab GTPase signaling in neurite outgrowth and axon specification. Cytoskeleton (Hoboken) 73:498-507.

Villarroel-Campos D, Henríquez DR, Bodaleo FJ, Oguchi ME, Bronfman FC, Fukuda M, Gonzalez-Billault C (2016b) Rab35 functions in axon elongation are regulated by P53-related protein kinase in a mechanism that involves Rab35 protein degradation and the microtubule-associated protein 1B. J Neurosci 36:7298-7313.

Wang T, Liu Y, Xu XH, Deng CY, Wu KY, Zhu J, Fu XQ, He M, Luo ZG (2011) Lgl1 activation of rab10 promotes axonal membrane trafficking underlying neuronal polarization. Dev Cell 21:431-444.

Yogev S, Shen K (2017) Establishing neuronal polarity with environmental and intrinsic mechanisms. Neuron 96:638-650. 